ARTICLE

\title{
The E3 ligase MREL57 modulates microtubule stability and stomatal closure in response to ABA
}

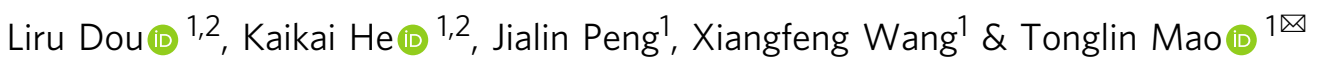

Regulation of stomatal movement is critical for plant adaptation to environmental stresses. The microtubule cytoskeleton undergoes disassembly, which is critical for stomatal closure in response to abscisic acid (ABA). However, the mechanism underlying this regulation largely remains unclear. Here we show that a ubiquitin-26S proteasome (UPS)-dependent pathway mediates microtubule disassembly and is required for ABA-induced stomatal closure. Moreover, we identify and characterize the ubiquitin E3 ligase MREL57 (MICROTUBULERELATED E3 LIGASE57) and the microtubule-stabilizing protein WDL7 (WAVE-DAMPENED2-LIKE7) in Arabidopsis and show that the MREL57-WDL7 module regulates microtubule disassembly to mediate stomatal closure in response to drought stress and $A B A$ treatment. MREL57 interacts with, ubiquitinates and degrades WDL7, and this effect is clearly enhanced by ABA. ABA-induced stomatal closure and microtubule disassembly are significantly suppressed in mrel57 mutants, and these phenotypes can be restored when WDL7 expression is decreased. Our results unravel UPS-dependent mechanisms and the role of an MREL57-WDL7 module in microtubule disassembly and stomatal closure in response to drought stress and ABA.

\footnotetext{
${ }^{1}$ State Key Laboratory of Plant Physiology and Biochemistry; Department of Plant Sciences, College of Biological Sciences, China Agricultural University, Beijing, China. ${ }^{2}$ These authors contributed equally: Liru Dou, Kaikai He. ${ }^{凶}$ email: maot12005@cau.edu.cn
} 
igher plants have developed finely tuned mechanisms to regulate gas exchange and water loss through stomata in leaves and stems. A pair of guard cells form stomatal pores and have developed intrinsic mechanisms to enable swelling or shrinkage, thus promoting the opening or closing of stomatal pores in response to environmental and stressful cues ${ }^{1}$. The stressrelated plant hormone abscisic acid (ABA) is a key signal inducing stomatal closure in response to drought stress. Multiple studies addressing $\mathrm{ABA}$-induced stomatal closure focus on $\mathrm{ABA}$ regulation of the activities of diverse membrane ion channels and transporters, such as $\mathrm{H}^{+}, \mathrm{K}^{+}$, and anion transporters and channels, through its receptor-phosphatase-kinase core signaling pathway, as well as the modulation of reactive oxygen species (ROS) and $\mathrm{Ca}^{2+}$ levels in the guard cells ${ }^{2-4}$. Other researchers have found that some ubiquitin E3 ligases participate in this physiological process. For example, a ubiquitin E3 ligase CHY ZINC-FINGER AND RING PROTEIN1 (CHYR1) promotes ABA-induced stomatal closure and its activity is regulated by Open Stomata 1 (OST1)/SnRK2.6mediated protein phosphorylation ${ }^{5}$. However, how those E3 ligases function and their underlying mechanisms in ABA-induced stomatal closure are largely unclear.

The organization and dynamics of the microtubule cytoskeleton are altered in response to diverse developmental cues and external signals, such as phytohormones, light, and salt stress, and are thus critical for the adaptation of plants to environmental and stressful conditions $^{6-12}$. Microtubule-associated proteins (MAPs) are the key regulators of microtubule dynamics, stability, and organization. Thus far, considerable progress has been made in understanding the molecular mechanism regarding how MAPs regulate microtubules to mediate plant cell growth in response to diverse signals 9 . For example, light signaling regulates the protein level of the MAP WAVE-DAMPENED2-LIKE3 (WDL3) to mediate hypocotyl cell elongation through the E3 ubiquitin ligase CONSTITUTIVE PHOTOMORPHOGENIC1 (COP1), which acts as a central repressor of seedling photomorphogenesis ${ }^{7}$. Although some studies with Vicia faba using the anti-microtubule drugs colchicine and paclitaxel suggested that microtubules have little impact on stomatal movement ${ }^{13}$; however, increasing evidence has produced conflicting results and support the notion that the microtubule cytoskeleton is vital for stomatal movement ${ }^{14-17}$. Pharmacological assays show that the microtubule-disrupting drug oryzalin inhibits stomatal opening, but that their closure is delayed by the microtubule-stabilizing drug paclitaxel ${ }^{14,15}$. Confocal imaging indicates that cortical microtubules undergo reorganizations during stomatal movement, including increasing assembly of cortical microtubules as stomata open and their disassembly as they close in response to ABA and darkness ${ }^{15-17}$. Although pharmacological evidence illustrates the critical impact of microtubules in stomatal movement, crucial genetic evidence is still lacking and the MAPs involved in stomatal movement are largely unidentified. In particular, much remains to be learned about the underlying regulatory mechanisms linking ABA and the MAP-microtubule modules and how they regulate stomatal closure in response to drought stress.

The ubiquitin-26S proteasome system (UPS) controls the degradation rates of numerous proteins and thus modulates multiple fundamental cellular and physiological processes, including plant growth, cell signaling, and stress responses ${ }^{18,19}$. The UPS consists of the concerted action of E1 Ub-activating enzymes, E2 Ub-conjugating enzymes, and E3 Ub ligases. The UPS leads to the covalent attachment of a multiubiquitin chain to target proteins, with the polyubiquitinated protein eventually being degraded by the $26 \mathrm{~S}$ proteasome ${ }^{19,20}$. Increasing evidence shows that the UPS regulates the organization and dynamics of cortical microtubules during the plant cell response to diverse signals. For example, the microtubule-stabilizing protein
SPIRAL1 is degraded by the UPS, which facilitates salt-induced microtubule disassembly and plant adaptation to high salinity conditions ${ }^{21}$. Khanna et al. ${ }^{22}$ showed that inhibition of proteolysis using the $26 \mathrm{~S}$ proteasome inhibitor MG132 impairs ABAinduced microtubule disassembly and stomatal closure, which suggests that UPS-dependent MAP degradation is required for ABA-induced microtubule disassembly and stomatal closure. Thus, identification of the MAPs regulated by the UPS and participating in ABA-induced stomatal closure will boost our understanding of the mechanisms underlying ABA-regulated plant environmental adaptation.

In this study, we demonstrate that the UPS is crucial for ABAinduced microtubule disassembly and stomatal closure. Furthermore, we functionally characterize an E3 ligase-MAP (MREL57-WDL7) module having an important role in stomatal closure and microtubule depolymerization in response to drought stress and ABA treatment.

\section{Results}

UPS-mediated microtubule disassembly is required for ABAinduced stomatal closure. A previous study showed that treatment with the 26S proteasome inhibitor MG132 suppressed ABA-induced microtubule disassembly and stomatal closure ${ }^{22}$, which led us to test whether the UPS has a role in regulating stomatal movement and microtubule stability in response to ABA. Thus, we chose $26 \mathrm{~S}$ proteasome mutant rpn1a-4 plants to investigate this hypothesis. Because ABA treatment results in the disassembly of cortical microtubules, we first determined microtubule stability in guard cells from rpn1a-4 mutant using the microtubule-disrupting drug oryzalin, and microtubule density was quantified as described previously by Higaki et al. ${ }^{23}$. When the stomata were in an open state, the microtubules exhibited well-organized radial filaments in both wildtype (WT) and rpn1a4 guard cells. Although the density of cortical microtubules was clearly decreased in the WT guard cells after 10-min oryzalin treatment, it was slightly reduced in rpn1a-4 guard cells. A longer duration resulted in the disappearance of most of the cortical microtubules in the guard cells in the WT but not rpn1a-4 seedlings (Fig. 1a, b; Supplementary Fig. 1a), indicating that $26 \mathrm{~S}$ proteasome-dependent proteolysis plays an important role in the regulation of microtubule stability in guard cells. In agreement with the results of oryzalin treatment, confocal imaging showed that cortical microtubules in guard cells from rpn1a-4 seedlings were less sensitive to ABA-induced microtubule disassembly (Fig. 1c, d; Supplementary Fig. 1b). Phenotype analysis showed that the ABA-induced stomatal closure evident in the WT was significantly blocked in the rpn1a-4 and rpn10-1 (another 26S proteasome mutant) mutants. Importantly, this blockage was partially restored by the exogenous application of oryzalin (Fig. 1e). This evidence thus demonstrates that the UPS promotes microtubule disassembly, which is required for $\mathrm{ABA}$-induced stomatal closure.

MAP WDL7 protein is degraded in guard cells in response to ABA. Given the central role of UPS-promoted microtubule disassembly in the ABA-induced stomatal closure, identification and characterization of the MAPs involved in these physiological processes will facilitate our understanding of the underlying mechanisms of stomatal movement in the response of plants to diverse signals. We found that the total YFP-tubulin fluorescence was clearly decreased in guard cells after ABA treatment for $40 \mathrm{~min}$. This decrease was owing to microtubule disassembly. Paclitaxel (Taxol) is a microtubule-stabilizing drug that promotes microtubule bundling and significantly suppresses microtubule disassembly $15,17,24,25$. We observed that the simultaneous treatment of guard cells with ABA 


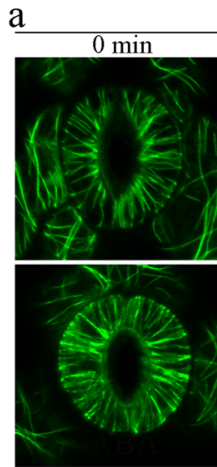

C

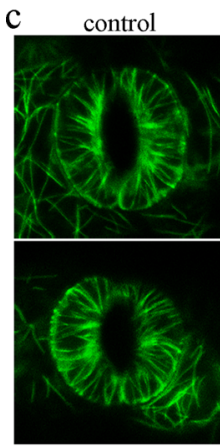
toryzalin

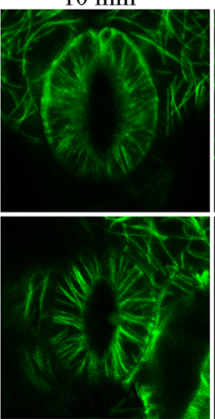

$\mathrm{ABA}$

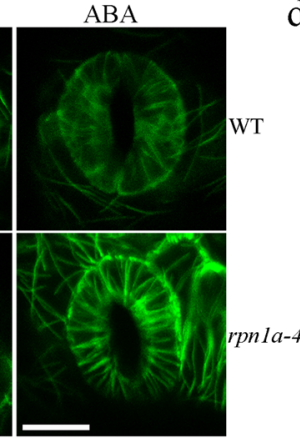

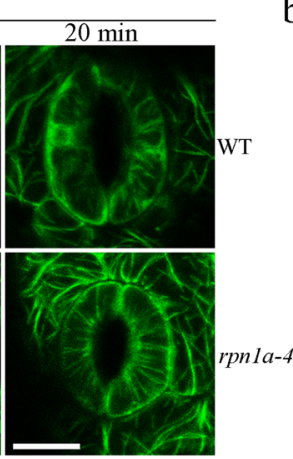

d
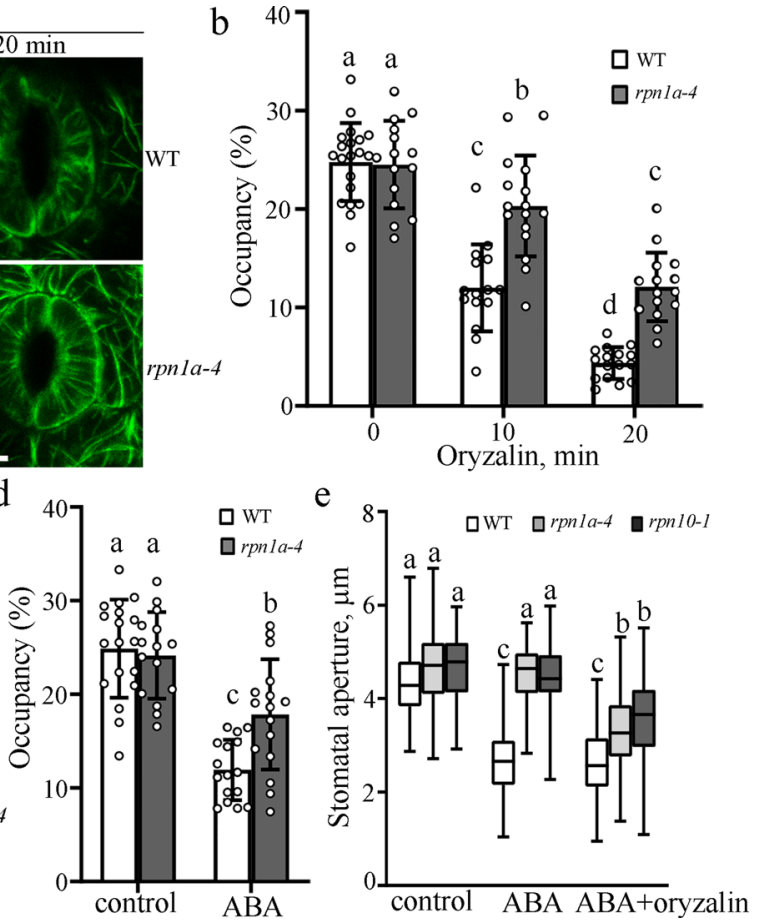

Fig. 1 Cortical microtubules in $\mathbf{2 6 S}$ proteasome mutant plants are insensitive to ABA-induced disassembly. a Cortical microtubules in guard cells from WT and rpn1a-4 seedlings treated with $5 \mu \mathrm{M}$ oryzalin for the indicated times. Scale bar $=10 \mu \mathrm{m}$. $\mathbf{b}$ The graph shows the densities of microtubules in $\mathbf{a}$. Data represent mean \pm standard deviation (SD) values from three independent experiments with a minimum of 15 cells each. c Cortical microtubules in guard cells from WT and rpn1a-4 seedlings treated with $10 \mu \mathrm{M}$ ABA for 40 min. Scale bar $=10 \mu \mathrm{m}$. $\mathbf{d}$ The graph shows the densities of microtubules in c. Data represent mean \pm standard deviation (SD) values from three independent experiments with a minimum of 15 cells each. e Detached rosette leaves from WT, rpn1a-4, and rpn10-1 were incubated in opening buffer for $2 \mathrm{~h}$ and then treated with $10 \mu \mathrm{M} \mathrm{ABA}$ plus 0 or $5 \mu \mathrm{M}$ oryzalin for $2 \mathrm{~h}$. The box and whiskers plots represent minimum and maximum values. The line in the box indicates the median value and the boundaries demonstrate the 25th percentile (upper) and the 75th percentile (lower). Different letters represent significant differences at $p<0.01$ (one-way ANOVA). The experiment was repeated three times as different biological replicates with a minimum of 100 stomatal pores.

and paclitaxel dramatically inhibited microtubule reduction and caused the microtubules to slightly bundle (Fig. 2a). Thus, we used this system to screen some members of WAVE-DAMPENED2 (WVD2)/WVD2-LIKE (WDL) family including WDL3, WDL4, WDL5, and WDL7, which we have focused on in recent years using GFP-tagged transgenic seedlings ${ }^{7,8,26,27}$. The total GFP signal was analyzed in the guard cells using an $\mathrm{ABA}$ stimulus as reference. We found that the total GFP fluorescence of a previously uncharacterized protein WDL7 was dramatically reduced in the guard cells from the WDL7-GFP transgenic seedlings following ABA treatment, even in the presence of paclitaxel. Furthermore, WDL7-GFP fluorescence was largely similar to the control condition in the guard cells when the 26S proteasome inhibitor MG132 was added into the system (Fig. 2b). In addition, WDL7-GFP protein levels were similar in the presence of oryzalin or paclitaxel alone (Supplementary Fig. 2). The GFP fluorescent spot color in Fig. $2 b$ indicated autofluorescence emitted by chloroplasts (Supplementary Fig. 3). In particular, the total GFP fluorescence of WDL7 was not that different in the pavement or root epidermal cells after ABA treatment (Fig. 2c, Supplementary Fig. $4 \mathrm{a}-\mathrm{c}$ ), demonstrating that the decreased WDL7GFP fluorescence in guard cells was not due to photobleaching. WDL7-GFP transgenic plants on a mCherry-tubulin background were further generated by crossing. In vivo time-course analysis showed that the microtubule disassembly preceded stomatal closure, and that degradation of WDL7-GFP temporally preceded microtubule disassembly and stomatal closure (Supplementary Fig. 5), implying that regulation of WDL7 protein level is critical for ABAinduced stomatal closure.

Then, we conducted cell-free degradation assays to confirm this degradation. Purified recombinant MBP-WDL7-FLAG protein from Escherichia coli was incubated with equal amounts of total protein extracted from ABA-treated and untreated WT seedlings in the presence of ATP. Immunoblot analysis showed that MBPWDL7-FLAG was degraded after an extended time of ATP application in the WT without ABA treatment. Impressively, this degradation was significantly increased in the ABA-treated WT but was largely blocked by the addition of MG132 (Fig. 2d, e). Next, WDL7 protein levels were analyzed using WDL7-GFP transgenic seedlings. Western blotting using an anti-GFP antibody showed that the WDL7-GFP levels in the leaves were clearly decreased in the presence of ABA but largely restored when the seedlings were treated with both ABA and MG132 (Fig. 2f, g). These findings confirm that WDL7 is degraded through the $26 \mathrm{~S}$ proteasome pathway during $\mathrm{ABA}$-induced stomatal closure.

Overexpression of WDL7 renders stomatal closure less sensitive to ABA. To investigate the in vivo function of WDL7 in ABA-induced stomatal closure, the levels of the WDL7 transcript were assessed using transgenic plants harboring $\beta$-glucuronidase (GUS) reporter gene under the control of the $\sim 2-\mathrm{kb}$ WDL7 promoter. Twelve independent transgenic lines were stained for GUS activity. GUS staining showed that WDL7 was widely expressed throughout the plant (Supplementary Fig. 6). Further analysis revealed that WDL7 was expressed in stomata, but the staining was similar in the presence of ABA (Fig. 3a). RNA was purified from the leaves of WT seedlings treated with ABA, and quantitative real-time PCR analyses were performed. WDL7 expression was largely similar after ABA treatment for $0,0.5,1$, and $2 \mathrm{~h}$ (Fig. $3 \mathrm{~b}$, left panel). The ABA-responsive gene RD29A was used as a positive control (Fig. $3 \mathrm{~b}$, right panel). These results 


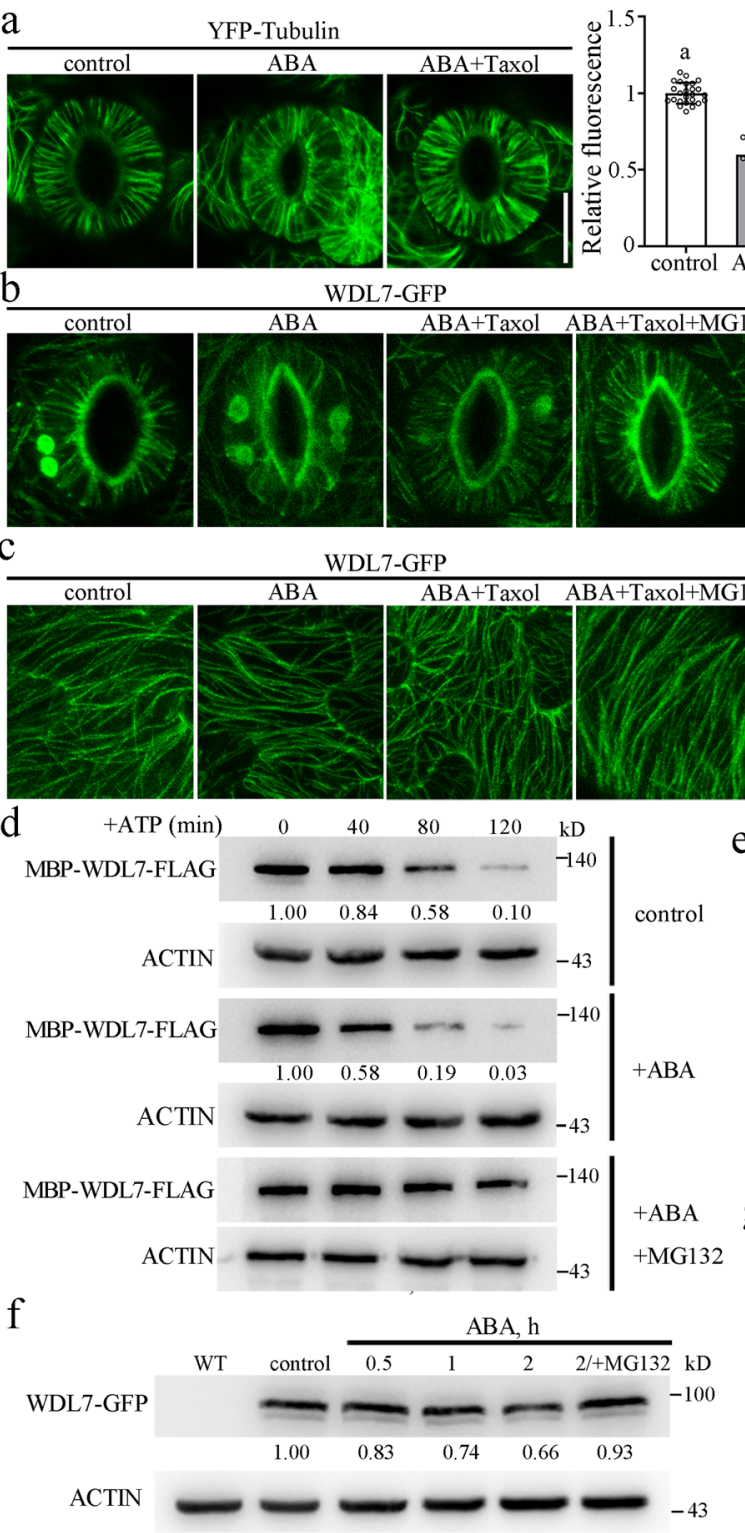

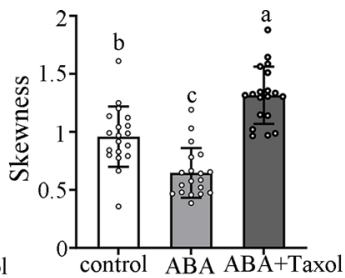
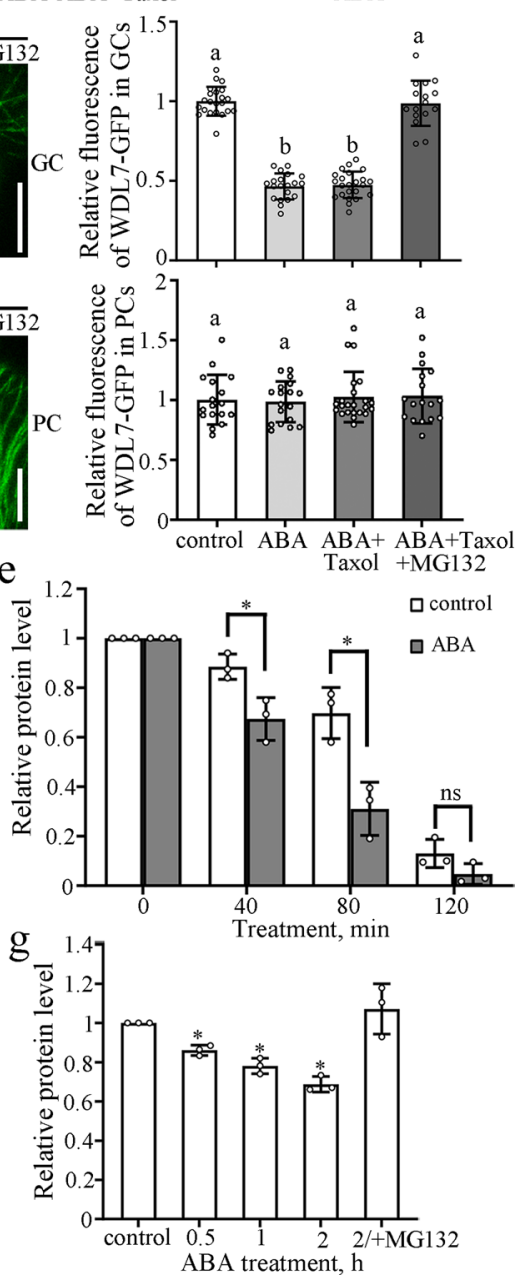

Fig. 2 WDL7 is degraded during ABA-induced stomatal closure. a Detached rosette leaves from YFP-tubulin were incubated in opening buffer for $2 \mathrm{~h}$ and then treated with $10 \mu \mathrm{M}$ ABA plus 0 or $20 \mu \mathrm{M}$ Taxol for $40 \mathrm{~min}$. Scale bar $=10 \mu \mathrm{m}$. The graphs show the relative fluorescence of YFP-tubulin and quantitative analysis of microtubule bundling (skewness). Data represent mean \pm standard deviation (SD) values from three independent experiments with a minimum of 15 cells each. Different letters represent significant differences at $p<0.01$ (one-way ANOVA). b, c Detached rosette leaves from WDL7-GFP transgenic seedlings were incubated in opening buffer for $2 \mathrm{~h}$ and then treated with $10 \mu \mathrm{M} \mathrm{ABA}, 10 \mu \mathrm{M}$ ABA plus $20 \mu \mathrm{M}$ Taxol, $10 \mu \mathrm{M}$ ABA plus $20 \mu \mathrm{M}$ Taxol and $50 \mu \mathrm{M}$ MG132 for $40 \mathrm{~min}$. Confocal images of the guard cells and pavement cells were taken. Scale bar $=10 \mu \mathrm{m}$. The graph shows the relative fluorescence of WDL7-GFP in $\mathbf{b}$ and $\mathbf{c}$. Data represent mean \pm standard deviation (SD) values from three independent experiments with a minimum of 15 cells each. Different letters represent significant differences at $p<0.01$ (one-way ANOVA). $\mathbf{d}$ Ten-day-old WT seedlings were treated with mock buffer, $10 \mu \mathrm{M} \mathrm{ABA}$, or $10 \mu \mathrm{M}$ ABA plus $50 \mu \mathrm{M}$ MG132 for $1 \mathrm{~h}$ and then total proteins were extracted. Purified MBP-WDL7-FLAG was incubated with equal amount of total proteins for the indicated times. MBP-WDL7-FLAG was detected with anti-FLAG antibody. Actin was used as a control. e Quantitative analysis of protein levels in $\mathbf{d}$. The WDL7 protein level at $0 \mathrm{~h}$ was set to 1 as a reference for calculating the relative protein levels at the various time points. Data represent the mean \pm SD for three independent experiments. Two-tailed Student's $t$ test, ${ }^{\star} p<0.05$. $\mathbf{f}$ Ten-day-old WDL7-GFP transgenic seedlings were treated with mock buffer, $10 \mu \mathrm{M}$ ABA, or $10 \mu \mathrm{M}$ ABA plus $50 \mu \mathrm{M} \mathrm{MG} 132$ for indicated times and then total proteins were extracted from the leaves. WDL7GFP was detected with anti-GFP antibody. Actin was used as a control. $\mathbf{g}$ Quantitative analysis of protein levels in $\mathbf{f}$. The protein level of WDL7-GFP treated with mock buffer was set to 1 as a reference for calculating relative protein levels of various time points. Data represent the mean \pm SD for three independent experiments. Two-tailed Student's $t$ test, ${ }^{\star} p<0.05$.

demonstrate that ABA does not affect the WDL7 transcriptional level.

To dissect the physiological role of WDL7 in ABA-induced stomatal closure, we obtained a $w d l 7-1$ T-DNA insertion mutant and created another mutant ( $w d l 7-2)$ using the CRISPR/Cas9 technique $^{28}$ (Supplementary Fig. 7). In addition, of 15 WDL7-GFPoverexpressing lines obtained, lines 1 and 2 were selected for further analysis. The WDL7 transcript level was dramatically reduced in the $w d l 7-1$ mutant and considerably increased in the overexpressing lines (OE\#1 and OE\#2, OE\#1 was used in the previous assay of the 

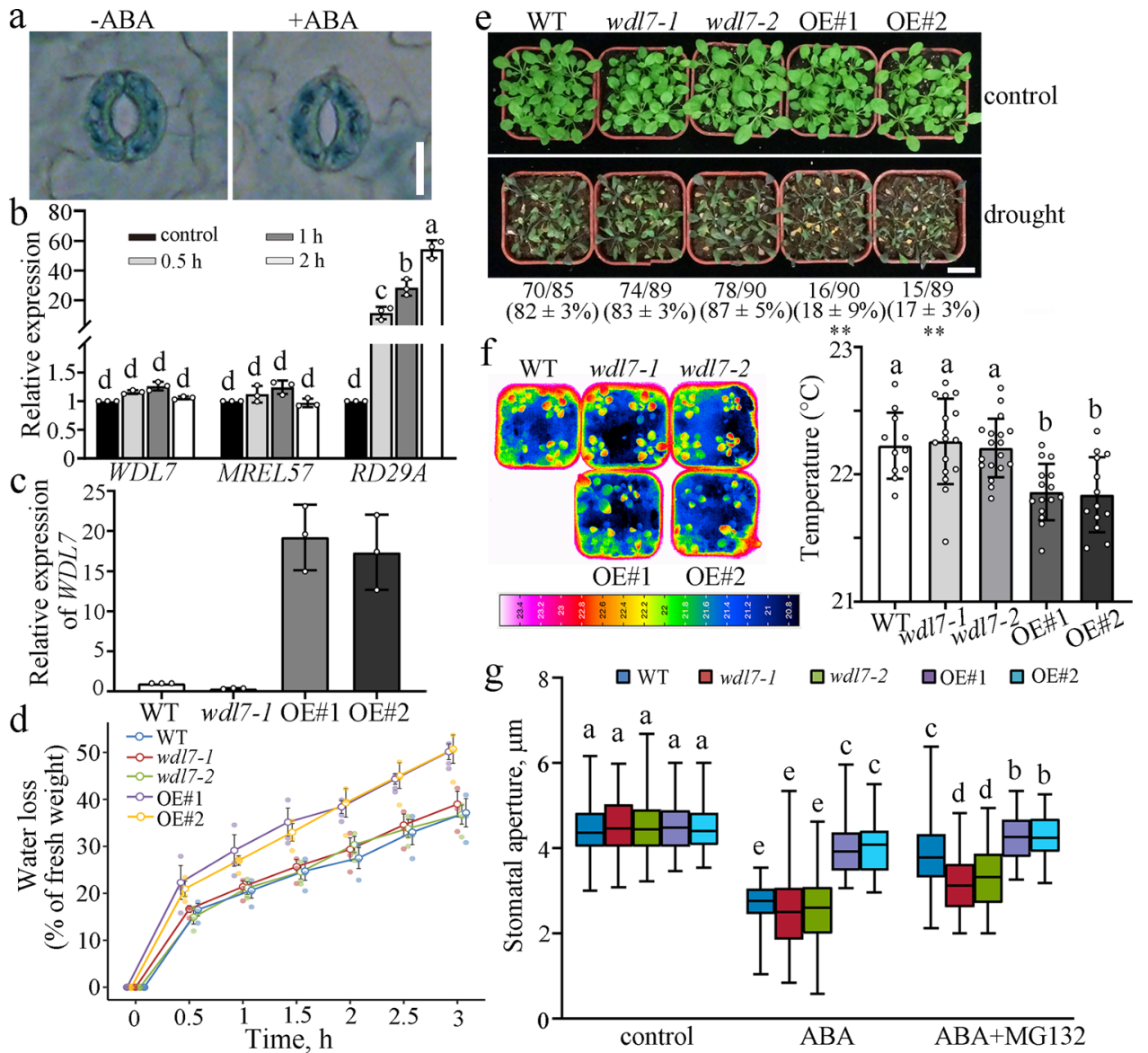

Fig. 3 Overexpression of WDL7 impairs the stomatal closure in response to ABA. a Detached rosette leaves from ProwDL7:GUS transgenic seedlings were incubated in opening buffer for $2 \mathrm{~h}$ and then treated with or without $10 \mu \mathrm{M}$ ABA for $2 \mathrm{~h}$. The epidermal strips were peeled from the rosette leaves and GUS staining was performed. Scale bar $=10 \mu \mathrm{m}$. b Quantitative real-time PCR analysis of WDL7 and MREL57 RNA levels in leaves after various treatment durations using $10 \mu \mathrm{M}$ ABA. UBQ11 was used as a reference gene and RD29A was a positive control. Gene expression levels in seedlings treated with mock buffer were set to 1 . The data represent the mean \pm SD for three independent experiments. Significant differences from corresponding untreated seedlings are indicated by different letters ( $p<0.01$ ), as determined by one-way ANOVA. c WDL7 expression was detected by quantitative real-time PCR in wdl7-1 mutant and two WDL7-overexpressing lines (OE\#1 and OE\#2). The data represent the mean \pm SD for three independent experiments. $\mathbf{d}$ Fresh weights of the detached leaves of seedlings from WT, wdl7-1, wdl7-2, OE\#1, and OE\#2 were measured at the indicated times. The experiment was repeated three times with independent treatments. Error bars indicate standard deviation. e Drought phenotypes of WT, wdl7-1, wdl7-2, OE\#1, and OE\#2 seedlings in soil. Three-week-old seedlings were subjected to drought stress by withholding water for two weeks before being photographed. Scale bar $=2 \mathrm{~cm}$. Values represent mean \pm SD for three independent experiments. Two-tailed Student's $t$ test, ${ }^{\star \star} p<0.01$. f Infrared thermography of seedlings from WT, wdl7-1, wdl7-2, OE\#1, and OE\#2. Images of 4-week-old plants in soil were taken using an infrared camera. The graph shows the leaf temperature measured using infrared camera software. Data represent mean \pm standard deviation (SD) values. Different letters represent significant differences at $p<0.01$ (one-way ANOVA). The experiment was repeated three times with independent treatments. $\mathbf{g}$ Detached rosette leaves of seedlings from WT, wdl7-1, wdl7-2, OE\#1, and OE\#2 were incubated in opening buffer for $2 \mathrm{~h}$ and then treated with $10 \mu \mathrm{M}$ ABA plus 0 or $50 \mu \mathrm{M} \mathrm{MG132}$ for another $2 \mathrm{~h}$. The box and whiskers plots represent minimum and maximum values. The line in the box indicates the median value and the boundaries demonstrate the 25th percentile (upper) and the 75th percentile (lower). Different letters represent significant differences at $p<0.01$ (one-way ANOVA). The experiment was repeated three times as different biological replicates with a minimum of 100 stomatal pores.

WDL7-GFP signal) (Fig. 3c). A water loss assay using the detached rosette leaves of 4-week-old plants showed that the rate of water loss was similar in $w d l 7$ mutants and in WT but was faster in WDL7GFP-overexpressing seedlings (OE\#1 and OE\#2) (Fig. 3d). We further compared their ability to avoid water loss by analyzing the difference in survival among all genotypes after soil drying. Consistent with the water loss results, there was no significant difference in the survival rates between the WT and wdl7-mutant seedlings. However, WDL7-GFP-overexpressing seedlings (OE\#1 and $\mathrm{OE} \# 2$ ) exhibited a hypersensitive phenotype with lower survival rates (Fig. 3e). In addition, an infrared camera was used to determine the transpiration rates of shoots by measuring the leaf temperature of 4-week-old plants. There was no significant difference in leaf temperature between $w d l 7$ mutants and WT.
However, leaf temperatures were significantly lower in OE\#1 and OE\#2 than in WT (Fig. 3f), suggesting that WDL7-GFPoverexpressing leaves display a higher transpiration rate and lose more water than WT.

Furthermore, a stomatal aperture assay was performed to determine the function of WDL7 in ABA-induced stomatal closure. Stomatal closure was clearly delayed in OE\#1 and OE\#2, whereas $w d l 7$ mutants showed no significant difference from WT in response to ABA (Fig. 3g, left side). Among WVD2/WDL family members, WDL3 and WDL4 are closely related to WDL $^{29}$. GUS staining indicated that WDL4 is not expressed in guard cells even after ABA treatment. WDL3 is expressed in guard cells (Supplementary Fig. 8a). Stomatal closure in wdl3-1 mutant was more sensitive to ABA treatment; however, WDL3-GFP 
protein was not degraded by this treatment (Supplementary Fig. $8 \mathrm{~b}, \mathrm{c}$ ). These evidence suggest that the lack of a significant phenotype in the $w d l 7$ mutants in response to ABA is because ABA-induced stomatal closing involves the degradation of WDL7 protein. Accordingly, the $26 \mathrm{~S}$ proteasome inhibitor MG132 partially repressed the sensitivity of stomatal closure to ABA in the WT seedlings, but the effect of MG132 was clearly inhibited in the $w d l 7$ mutants. For the WDL7-GFP-overexpressing seedlings

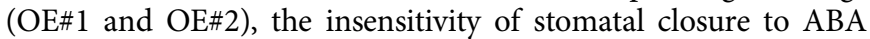
was enhanced when treated with both ABA and MG132 (Fig. 3g, right side). Taken together, these results demonstrated that WDL7 has a negative role in $26 \mathrm{~S}$ proteasome degradation pathwaymediated stomatal closure, supporting the notion that WDL7 degradation via the $26 \mathrm{~S}$ proteasome degradation pathway is essential for stomatal closure in response to drought stress and ABA treatment.
WDL7 is a microtubule stabilizer. Given that cortical microtubule destabilization is required for ABA-induced stomatal closure, we investigated the molecular basis for the WDL7 regulation of microtubules. We observed the subcellular localization of WDL7 in the guard cells using WDL7-GFP transgenic plants on an $m$ Cherry-tubulin background. Confocal microscopy showed that the WDL7-GFP fluorescence overlapped the red fluorescent signal of cortical microtubules (Fig. 4a), even in the presence of ABA, ABA plus Taxol, or MG132 (Supplementary Fig. 9a). Colocalization was analyzed by plotting the signal intensities of WDL7-GFP and microtubules using ImageJ software (Fig. 4b). The results demonstrated that WDL7 localized to microtubules in the guard cells. Moreover, confocal imaging showed that HisWDL7-GFP fusion protein directly bound to and bundled paclitaxel-stabilized microtubules that were polymerized from rhodamine-labeled tubulins (Fig. 4c, d), which is similar to other
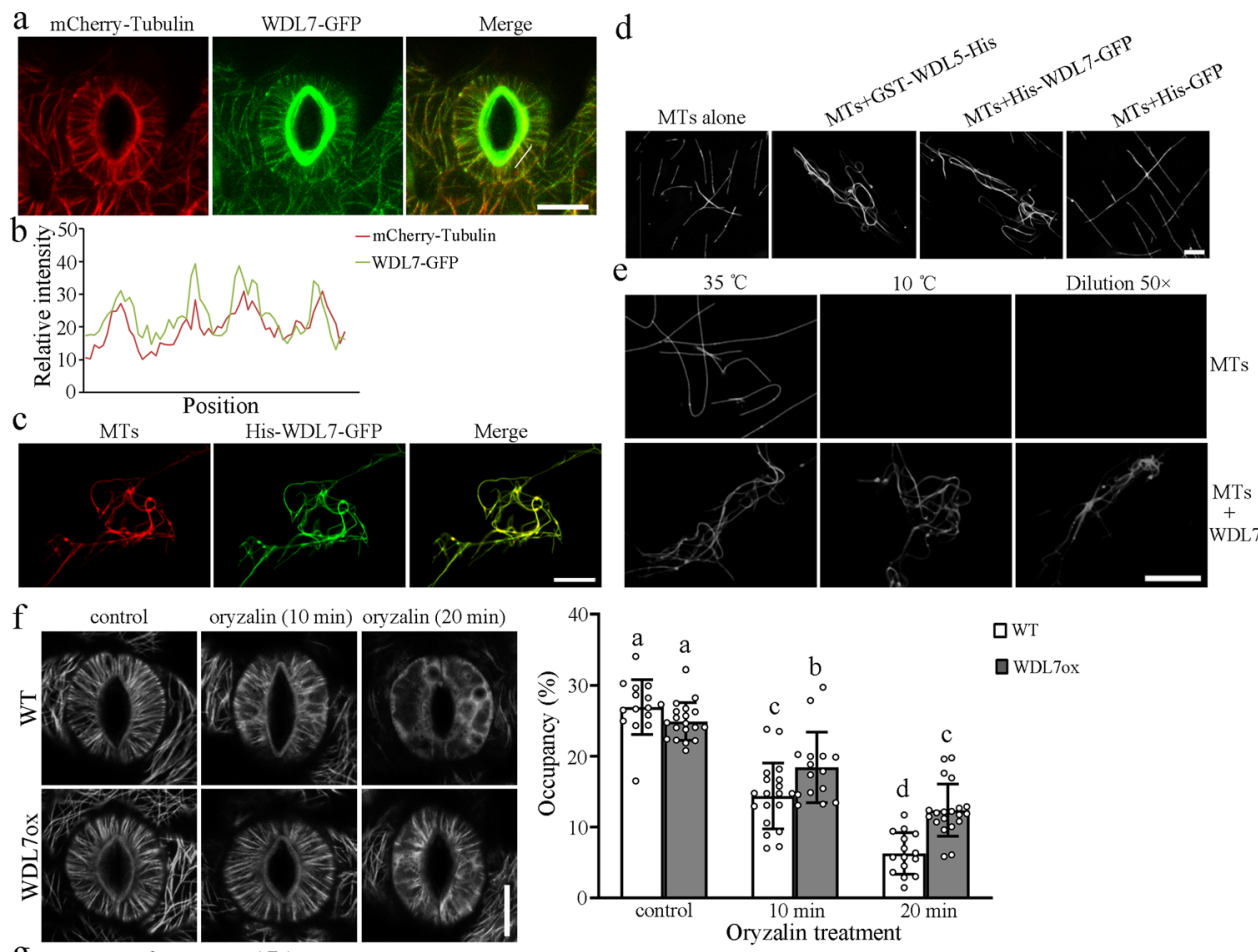

口WT
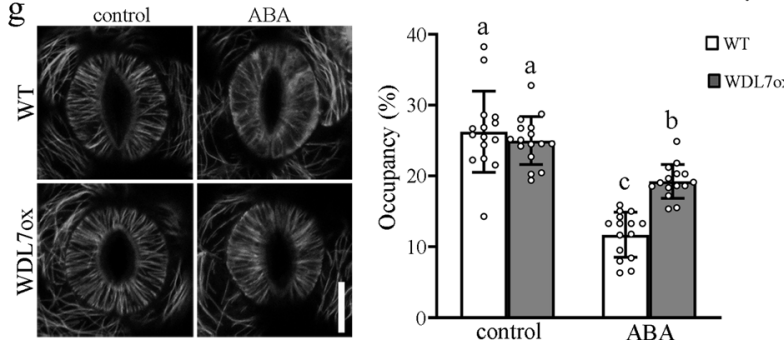

Fig. 4 WDL7 binds to and stabilizes microtubules in vitro and in vivo. a WDL7-GFP colocalizes with cortical microtubules detected in guard cells. Detached rosette leaves of seedlings from WDL7-GFP transgenic plants on an mCherry-tubulin background were incubated in opening buffer for $2 \mathrm{~h}$. The experiment repeated three times with similar results. Scale bar $=10 \mu \mathrm{m}$. b Plot of a line scan drawn in a showing a strong correlation between the spatial localization of WDL7-GFP and cortical microtubules. c His-WDL7-GFP colocalizes with microtubules polymerized from rhodamine-labeled tubulin in vitro. The experiment repeated three times with similar results. Scale bar $=10 \mu \mathrm{m}$. $\mathbf{d}$ WDL7 induced the formation of microtubule bundles in vitro. Images show microtubules polymerized from rhodamine-labeled tubulin incubated with $3 \mu \mathrm{M}$ His-WDL7-GFP, GST-WDL5-His, or His-GFP protein for $30 \mathrm{~min}$. The experiment repeated three times with similar results. Scale bar $=5 \mu \mathrm{m}$. e WDL7 stabilizes microtubules against cold and dilution-induced depolymerization. The experiment repeated three times with similar results. Scale bar $=10 \mu \mathrm{m}$. f Cortical microtubules in guard cells from WT and WDL7GFP transgenic seedlings (WDL7ox) on an mCherry-tubulin background treated with $5 \mu \mathrm{M}$ oryzalin for 10 and $20 \mathrm{~min}$. Scale bar $=10 \mu \mathrm{m}$. The graph shows the densities of microtubules in $\mathbf{f}$. Data represent mean \pm standard deviation (SD) values from three independent experiments with a minimum of 15 cells each. $\mathbf{g}$ Cortical microtubules in guard cells from WT and WDL7-GFP transgenic seedlings (WDL7ox) on an mCherry-tubulin background treated with 0 and $10 \mu \mathrm{M}$ ABA for $40 \mathrm{~min}$. Scale bar $=10 \mu \mathrm{m}$. The graph shows the densities of microtubules in $\mathbf{g}$. Data represent mean \pm standard deviation (SD) values for three independent experiments with a minimum of 15 cells each. Different letters represent significant differences at $p<0.01$ (one-way ANOVA). 
members from the WVD2/WDL family such as WDL3 and WDL5 $5^{26,27}$.

The effects of WDL7 on microtubule stability were further investigated using cold and dilution treatments that can rapidly induce microtubule depolymerization ${ }^{30}$. Microtubules polymerized from rhodamine-labeled tubulin $(20 \mu \mathrm{M})$ were incubated in the presence and absence of $3 \mu \mathrm{M}$ His-WDL7-GFP (Fig. 4e, left panels). Then, the solutions were incubated at $10^{\circ} \mathrm{C}$ for $30 \mathrm{~min}$ (Fig. $4 \mathrm{e}$, middle panels) or diluted with $50 \times$ prewarmed buffer and incubated at $35^{\circ} \mathrm{C}$ for $60 \mathrm{~min}$ (Fig. 4e, right panels). Confocal microscopy data showed that microtubule filaments were fully disassembled in the absence of WDL7, but that many microtubules persisted in the presence of WDL7 after the cold and dilution treatments. These results demonstrate that WDL7 can stabilize microtubules against low temperature- and dilutioninduced depolymerization.

In order to characterize the effect of WDL7 on cortical microtubules in vivo, the microtubule-disrupting drug oryzalin was applied to guard cells against WT and WDL7-GFPoverexpressing seedlings. When the stomata were in an open state, the microtubules exhibited well-organized radial filaments in both the WT and WDL7-GFP-overexpressing guard cells. However, cortical microtubules were disrupted in the guard cells from WT after treatment with $5 \mu \mathrm{M}$ oryzalin for $10 \mathrm{~min}$, whereas the microtubules in WDL7-GFP-overexpressing guard cells were largely unaffected. Increases in the duration of oryzalin treatment resulted in disruption of the majority of cortical microtubules in WT but not WDL7-GFP-overexpressing guard cells (Fig. 4f; Supplementary Fig. 1c). Thus, microtubules were less sensitive to oryzalin treatment when the expression of WDL7 was increased. In agreement with the above results, most of the cortical microtubules disappeared after ABA treatment in the WT guard cells, whereas many microtubules were observed in WDL7-GFPoverexpressing guard cells (Fig. 4g; Supplementary Fig. 1d). Previous studies showed that increased polymerization of the cortical microtubules is observed when stomata opening is induced by light ${ }^{15}$. We found that WDL7 was expressed in guard cells in both the dark and light, and the light-induced stomatal opening was partially suppressed in the wdl7 mutants (Supplementary Fig. 10a, b). Accordingly, microtubule assembly was partially suppressed in $w d l 7$-mutant guard cells in response to light compared with WT (Supplementary Fig. 10c). These results confirm that WDL7 functions as a microtubule stabilizer.

An E3 ligase MREL57 interacts with and ubiquitinates WDL7. Given that WDL7 is degraded in response to ABA, we used WDL7 as the bait to screen for potential interacting proteins in a yeast two-hybrid screen. Six independent clones were isolated, which encoded deletion products of a potential E3 ligase. We named this gene MREL57 (Microtubule-related E3 Ligase 57), which contains coiled-coil and RING domains in the C-terminal region (Fig. 5a). MREL57 is classified in cluster 2.3 according to cluster analysis and the metal-ligand arrangement of the RING domain $^{31}$. The RING domain of MREL57 is highly conserved with well-characterized E3 ligases in cluster 2.3 (Supplementary Fig. 11a). An in vitro ubiquitination assay using an MBPMREL57-MYC fusion protein showed that MREL57 was capable of autoubiquitination, as detected by anti-Ub and anti-MYC antibodies. When we mutated conserved amino acid in the RING-H2 finger domain (Cys-486 residue was replaced with Ala486), which is responsible for binding to $\mathrm{Zn}^{2+}$, the MBPMREL57 ${ }^{\mathrm{C} 486 \mathrm{~A}}$-MYC lost its E3 ligase activity (Supplementary Fig. 11b). These findings demonstrate that MREL57 is a new E3 ligase and that the RING-H2 domain is essential for its E3 ligase activity.
Our yeast two-hybrid assay data revealed that WDL7 interacted with full-length MREL57, but not with the known E3 ligases in cluster 2.3, including JUL1, MBR2, TEAR1, and TEAR2 (Fig. 5b, Supplementary Fig. 11c). In addition, MREL57 was unable to interact with either WDL3 or WDL4 in the yeast (Supplementary Fig. 11d). Previous studies showed that many members in WVD2/ WDL family-like WDL3 and WDL5 participate in hypocotyl cell elongation $7,26,27$. Although $W D L 7$ was also expressed in the hypocotyls, no obvious hypocotyl-elongated phenotype of $w d l 7$ mutants was found in the light- and dark-grown seedlings (Supplementary Fig. 12). Thus, these results reveal the diverse physiological roles of WVD2/WDL proteins, and imply a specific MREL57-WDL7 module function in ABA-induced stomatal closure. Deletion experiments showed that WDL7 interacted with the MREL57 $\mathrm{N}$ terminus, but not with the $\mathrm{C}$ terminus (Fig. 5b). To further investigate the MREL57-WDL7 interaction, we generated MREL57-GFP transgenic plants driven by the UBQ10 promoter and performed a semi-in vivo pull-down assay. The results showed that MBP-WDL7-FLAG, but not MBP, could successfully pull-down MREL57 in Arabidopsis (Fig. 5c). Furthermore, the in vivo interaction of WDL7 and MREL57 was validated by a firefly luciferase complementation imaging assay in tobacco leaves ${ }^{32}$. Although no reconstituted luminescence was detected when MREL57 and WDL7 were co-expressed in the absence of MG132, strong luminescence signals appeared in the presence of MG132 or when MREL57 was replaced with mutated MREL57 (MREl57 C486A) (Fig. 5d). These results further confirmed that MREL57 interacts with and degrades WDL7. Moreover, we found that the interaction of MREL57 (using MREl57 ${ }^{\mathrm{C} 486 \mathrm{~A}}$ ) and WDL7 was significantly enhanced by ABA treatment (Fig. $5 \mathrm{e}-\mathrm{g}$ ).

The physical interaction between MREL57 and WDL7 prompted us to test whether WDL7 is a substrate of the MREL57 E3 ligase using an in vitro ubiquitination assay. MBP-WDL7FLAG protein was purified from E. coli as substrate and MBPMREL57-MYC recombinant protein acts as the E3 ligase. In the presence of E1, E2, and ubiquitin, MBP-WDL7-FLAG was polyubiquitinated by MBP-MREL57-MYC, as detected by anti$\mathrm{Ub}$ and anti-FLAG antibodies (Fig. 5h). Furthermore, we examined the ubiquitination of WDL7 in the WDL7-GFPoverexpressing plants with or without ABA treatment. The polyubiquitination of WDL7 was detected with anti-Ub antibody, and the level of polyubiquitinated WDL7 was substantially increased by ABA treatment (Fig. 5i, Lanes 1 and 2; 5j, left panel). These results illustrate that WDL7 is a substrate of the MREL57 E3 ligase and that ABA enhances the MREL57 interaction with and ubiquitination of WDL7.

MREL57 destabilizes microtubules and positively regulates stomatal closure in response to ABA. Given that MREL57 interacts with and ubiquitinates WDL7, we proposed that MREL57 has a role in regulating microtubule stability and stomatal closure in response to drought stress and $\mathrm{ABA}$ treatment. GUS staining of transgenic seedlings carrying $\operatorname{Pro}_{\text {MREL57:GUS indicated that }}$ MREL57 was constitutively expressed in most tissues (Supplementary Fig. 13). Furthermore, MREL57 was expressed in stomata, with no clear difference after ABA treatment (Fig. 6a). Real-time PCR further confirmed that MREL57 expression was not regulated by ABA treatment (Fig. 3b, middle panel). We obtained two TDNA insertion mutants (mrel57-1 and mrel57-2) for MREL57. Real-time qPCR analysis determined that the transcript accumulation of MREL57 was significantly decreased in mrel57-1 and mrel57-2 mutants (Fig. 6b). A water loss assay showed that the water loss rate of detached leaves was faster in mrel57 mutants than in WT (Fig. 6c). Accordingly, the mrel57 mutant seedlings were more sensitive to drought stress and leaf temperatures were 


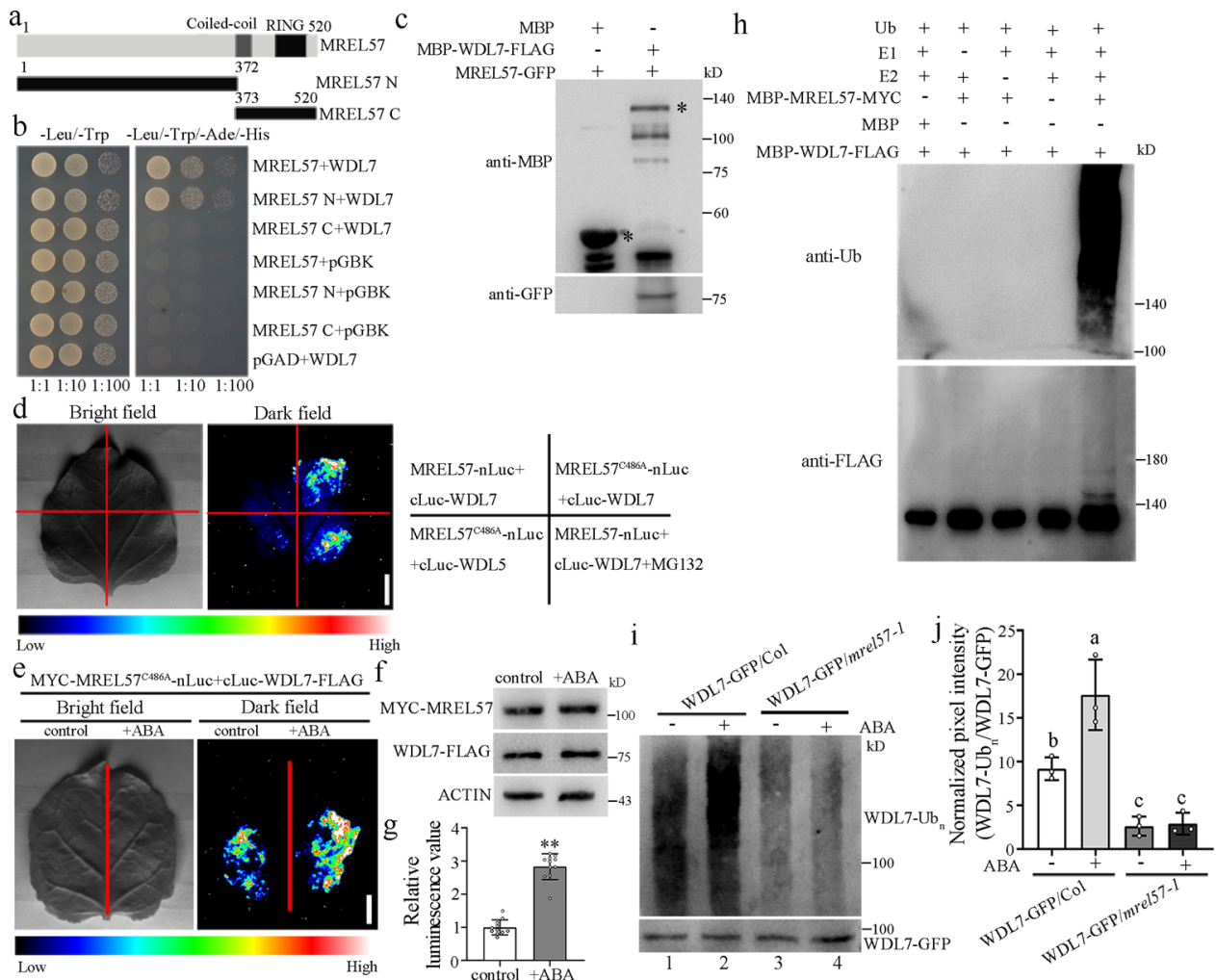

Fig. 5 The E3 ligase MREL57 interacts with and ubiquitinates WDL7. a Schematic representation of MREL57 truncated proteins. b WDL7 interacts with MREL57 and MREL57 $\mathrm{N}$ in a yeast two-hybrid assay. c A semi-in vivo pull-down assay showed an interaction between MREL57 and WDL7. MBP-WDL7FLAG and MBP were marked with asterisks, respectively. The experiment repeated three times with similar results. $\mathbf{d}$ Split-luciferase complementation assay to analyze the interaction between MREL57 and WDL7 in N. benthamiana leaves. MG132 at $50 \mu \mathrm{M}$ was injected into the leaf tissues $12 \mathrm{~h}$ before imaging. The pseudo-color bar shows the range of luminescence. Scale bar $=1 \mathrm{~cm}$. e ABA enhances the interaction between MREL57 and WDL7. MYCMREL57C486A-nluc and cluc-WDL7-FLAG were transformed into N. benthamiana leaves. After a 2-day incubation, the leaves were treated with mock buffer or $100 \mu \mathrm{M}$ ABA for $2 \mathrm{~h}$. Scale bar $=1 \mathrm{~cm}$. f WDL7 and MREL57 protein levels were detected by western blotting using anti-FLAG and anti-MYC antibodies. Actin was used as a control. $\mathbf{g}$ Luminescence values were determined using a spectrophotometer. The experiment was repeated three times. Data represent mean \pm standard deviation (SD) values. Two-tailed Student's $t$ test, ${ }^{\star \star} p<0.01$. h MREL57 ubiquitinates WDL7 in vitro. Recombinant proteins were purified from E. coli and then incubated at $30^{\circ} \mathrm{C}$ for $3 \mathrm{~h}$. The ubiquitination of WDL7 was detected with anti-Ub and anti-FLAG antibodies. The experiment repeated three times with similar results. i MREL57 ubiquitinates WDL7 in vivo. WDL7-GFP and WDL7-GFP/mrel57-1 transgenic seedlings were treated with 0 or $10 \mu \mathrm{M}$ of ABA for $1 \mathrm{~h}$ in the presence of $50 \mu \mathrm{M}$ MG132. Total proteins were extracted and incubated with anti-GFP mAb-magnetic agarose. Anti-Ub was used to detect the polyubiquitination of WDL7. $\mathbf{j}$ Quantitative analysis of the signal intensity in $\mathbf{i}$. Data represent the mean \pm SD for three independent experiments. Different letters represent significant differences at $p<0.01$ (one-way ANOVA).

significantly lower in mrel57 mutants than in WT (Fig. 6d, e), suggesting that mrel57 mutant leaves display a higher transpiration rate and lose more water than the WT. The stomatal aperture assay showed that stomatal closure was less sensitive to ABA treatment in mrel57 mutants compared with WT (Fig. 6f, left and middle panels). This evidence demonstrates that MREL57 participates in promoting stomatal closure in response to drought stress and $\mathrm{ABA}$ treatment.

Next, we further investigated how MREL57 regulates microtubule stability during ABA-induced stomatal closure. We found that disruption of microtubules with oryzalin in the mrel57 mutant partially restored stomatal closure under ABA treatment (Fig. 6f, right panel), suggesting that microtubules are more stable in mrel57 guard cells. To test this hypothesis, we crossed mrel57-1 mutant with YFP-tubulin plants. Confocal microscopy showed that the microtubules exhibited well-organized radial filaments in both the WT and mrel57-1 guard cells on a YFP-tubulin background when the stomata were in an open state. After oryzalin treatment for $10 \mathrm{~min}$, many microtubules were disrupted in the WT guard cells, but not in the mrel57-1 guard cells. After a longer treatment, most of the microtubules disappeared in WT guard cells, but more microtubules were observed in mrel57-1 guard cells (Fig. 6g, Supplementary Fig. 1e). In agreement with the results of oryzalin treatment, microtubules mostly disassembled in the WT guard cells but persisted in the mrel57-1 guard cells after ABA treatment for $40 \mathrm{~min}$ (Fig. 6h, left panel, Supplementary Fig. 1f). Taken together, these results demonstrate that MREL57 promotes microtubule disassembly to mediate ABA-induced stomatal closure.

In addition, the water loss, leaf temperature, and stomatal closure phenotypes of mrel57 mutants were fully rescued by MREL57-GFP (Com\#1 and Com\#2), but not by mutated

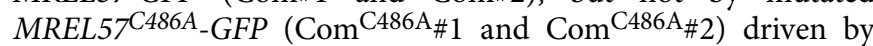
the native MREL57 promoter (Supplementary Fig. 14), demonstrating that the phenotype is indeed caused by the loss of MREL57 function and that the function of MREL57 depends on its E3 ligase activity. These results support the notion that MREL57 is required for ABA-induced stomatal closure by destabilizing microtubules.

MREL57 is responsible for WDL7 degradation in ABA-induced stomatal closure and microtubule disassembly. MREL57 interacts with the microtubule-stabilizing protein WDL7 and destabilizes 

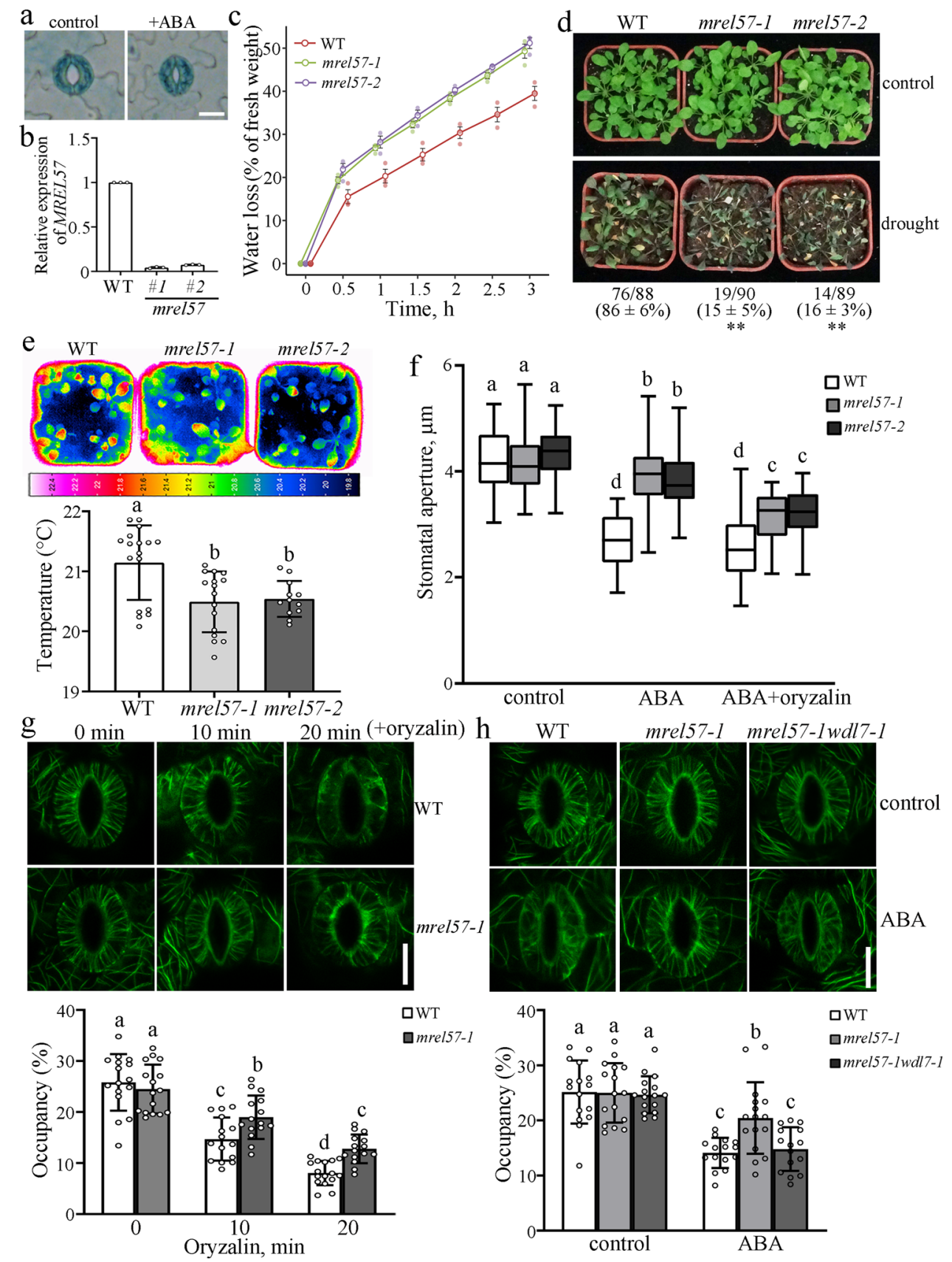

Fig. 6 MREL57 destabilizes microtubules and positively regulates stomatal closure in response to ABA. a Detached rosette leaves from Pro ${ }_{M R E L 57: G U S}$ transgenic seedlings were incubated in opening buffer for $2 \mathrm{~h}$ and then treated with or without $10 \mu \mathrm{M}$ ABA for $2 \mathrm{~h}$. The epidermal strips were peeled from the rosette leaves and GUS staining was performed. The experiment repeated three times with similar results. Scale bar $=10 \mu \mathrm{m}$. b MREL57 expression was detected by quantitative real-time RT-PCR in WT, mrel57-1, and mrel57-2 mutant seedlings. The experiment was repeated three times as different biological replicates. Error bars indicate standard deviation. c Fresh weights of detached leaves of seedlings from WT, mrel57-1, and mrel57-2 were measured every $30 \mathrm{~min}$ for a total of $3 \mathrm{~h}$. The experiment was repeated three times with independent treatments. d Drought phenotypes in the wildtype, mrel57-1, and mrel57-2 in soil. Three-week-old seedlings were subjected to drought stress by withholding water for 2 weeks before being photographed. Values represent mean \pm SD for three independent experiments. Two-tailed Student's $t$ test. ${ }^{\star \star} p<0.01$. e Infrared thermography of seedlings from WT, mrel57-1, and mrel57-2. Leaf temperature was measured using infrared camera software. The experiment was repeated three times with independent treatments. Error bars indicate standard deviation. Data represent mean \pm standard deviation (SD) values. Different letters represent significant differences at $p<0.01$ (one-way ANOVA). f Detached rosette leaves from WT, mrel57-1, and mrel57-2 were incubated in opening buffer for $2 \mathrm{~h}$ and then treated with $10 \mu \mathrm{M} A B A$ with or without oryzalin. The box and whiskers plots represent minimum and maximum values. The line in the box indicates the median value and the boundaries demonstrate the 25th percentile (upper) and the 75th percentile (lower). Different letters represent significant differences at $p<0.01$ (one-way ANOVA). The experiment was repeated three times as different biological replicates with a minimum of 100 stomatal pores. $\mathbf{g}$ Cortical microtubules in guard cells from WT and mrel57-1 seedlings treated with $5 \mu \mathrm{M}$ oryzalin for the indicated times. Scale bar $=10 \mu \mathrm{m}$. The graph shows the densities of microtubules. Data represent mean \pm standard deviation (SD) values from three independent experiments with a minimum of 15 cells each. $\mathbf{h}$ Cortical microtubules in guard cells from WT, mrel57-1 mutant, and mrel57-1wdl7-1 double mutant seedlings treated with $10 \mu \mathrm{M}$ ABA for 40 min. Scale bar $=10 \mu \mathrm{m}$. The graph shows the densities of microtubules. Data represent mean \pm standard deviation (SD) values from three independent experiments with a minimum of 15 cells each. 
microtubules to promote stomatal closure under ABA treatment, which led us to investigate whether MREL57 mediates the degradation of WDL7 during ABA-induced stomatal closure. We crossed the WDL7-GFP transgenic plant with mrel57-1 mutant to examine the protein level of WDL7-GFP on an mrel57-1 mutant background. Paclitaxel was used to stabilize microtubules under ABA treatment. When the stomata were in an open state, WDL7GFP exhibited well-organized radial filaments in both WDL7-GFPoverexpressing WT and mrel57-1 guard cells. After ABA plus paclitaxel treatment for $40 \mathrm{~min}$, the total WDL7-GFP fluorescence was dramatically reduced in the guard cells but not in pavement cells from the WDL7-GFP-overexpressing seedlings. However, WDL7-GFP signal remained largely unaffected in WDL7-GFP/ mrel57-1 seedlings (Fig. 7a, b). In addition, most of the WDL7-GFP colocalized to the microtubules in the mrel57 mutant guard cells in the presence of ABA and paclitaxel (Supplementary Fig. 9b). These results suggested that MREL57 mediates WDL7 degradation during ABA-induced stomatal closure.

Next, we conducted a cell-free degradation assay. Purified recombinant MBP-WDL7-FLAG protein was incubated with equal amounts of total protein extracted from WT and mrel57-1 seedlings in the presence of ATP. Immunoblot analysis showed that MBP-WDL7-FLAG was degraded after an extended period of ATP application in both the WT and mrel57-1 mutant; however, this degradation was much slower in the mrel57-1 mutant, even when these seedlings were treated with ABA (Fig. 7c, d). WDL7GFP transgenic WT and mrel57-1 seedlings were then treated with $100 \mu \mathrm{M}$ cycloheximide (CHX), a protein synthesis inhibitor, to further examine the effect of MREL57 on WDL7 degradation in response to ABA in vivo. We found that, although MREL57 could coimmunoprecipitate with WDL7 in both the leaves and roots, WDL7-GFP protein levels were only decreased in the leaves after ABA treatment (Fig. 2f, Supplementary Fig. 4d, e), suggesting that the MREL57-WDL7 module might mainly function in the leaves in response to ABA treatment. Accordingly, western blotting showed that the degradation of WDL7-GFP in leaves was clearly reduced in the mrel57-1 mutant compared with the WT, and this degradation was largely suppressed by MG132 (Fig. 7e, f). Consistent with this result, ABA-induced WDL7 ubiquitination was also significantly reduced in the mrel57-1 mutant (Fig. 5i, Lanes 3 and 4; 5j, right panel). Based on these combined results, we conclude that MREL57 is responsible for WDL7 degradation during $\mathrm{ABA}$-induced stomatal closure.

To elucidate the genetic interactions of MREL57 and WDL7, we generated mrel57-1wdl7-1 double mutants (mrel57-1wdl7-1\#1 and $m$ rel57-1wdl7-1\#2) by crossing. A water loss assay showed faster water loss in detached leaves of mrel57-1 mutant, but not in those of mrel57-1wdl7-1 double mutants, which exhibited a similar water loss phenotype to WT and wdl7-1 mutant (Fig. $7 \mathrm{~g}$ ). Similarly, there was no significant difference in leaf temperature between mrel57-1wdl7-1 double mutants from WT and $w d l 7-1$ mutant (Fig. 7h). Importantly, the insensitivity of ABA-induced stomatal closure and microtubule disassembly phenotypes in mrel57-1 mutant were clearly suppressed in mrel57-1wdl7-1 double mutants (Fig. 6h, right panels; 7i). Taken together, these results support the idea that MREL57-mediated WDL7 degradation is essential for microtubule disassembly and stomatal closure in response to drought stress and ABA treatment.

\section{Discussion}

In this study, we show that the UPS-mediated stability of cortical microtubules is critical for ABA-induced stomatal closure. Furthermore, MREL57-WDL7 module was identified to participate in microtubule disassembly and stomatal closure in response to drought stress and ABA treatment. Our results shed light on microtubule reorganization in guard cells in response to ABA and suggest a hypothetical model for MREL57-WDL7 function in ABA-induced stomatal closure (Fig. 8).

Cortical microtubules in guard cells undergo dynamic reorganization during stomatal movement. We found that microtubule disassembly preceded stomatal closure, which behavior was similar to that observed with microtubule reorganization and hypocotyl elongation in response to light and hormones ${ }^{11}$, supporting the notion that microtubule regulation facilitates stomatal movements. Microtubules are destabilized when stomata closure is induced by $\mathrm{ABA}$ and dark conditions, and exogenous application of the microtubule-stabilizing drug paclitaxel partially blocks stomatal closure, suggesting that the regulation of cortical microtubule stability is important for proper stomatal closure $^{15,16}$. In this study, we provided several lines of evidence supporting the hypothesis that cortical microtubule destabilization is required for ABA-induced stomata closure. Cortical microtubules are more stable in rpn1a-4 and mrel57 guard cells, and the microtubule-disrupting drug oryzalin partially restores the lower sensitivity of ABA-induced rpn1a-4 and mrel57 stomatal closures. WDL7, as a microtubule-stabilizing protein, has a negative role in UPS-regulated ABA-mediated microtubule disassembly and stomatal closure. Lower WDL7 expression clearly restored the lower sensitivity of mrel57 mutant to ABAinduced microtubule disassembly and stomatal closure. Our findings are in agreement with previous studies showing that ABA-induced stomatal closure and cortical microtubule destabilization were impaired in the E3 ligase CONSTITUTIVE PHOTOMORPHOGENIC 1 (COP1) mutant ${ }^{22}$. Besides WDL7, the MAPs WDL3 and SPR1 also function as microtubule-stabilizing proteins and are regulated by the UPS ${ }^{9}$. Taken together, these studies, therefore, suggested that the UPS mainly induces microtubule destabilization through degradation of diverse microtubulestabilizing proteins, which is required for multiple UPS-mediated physiological processes, including ABA-induced stomatal closure. Future studies will provide more experimental evidence to address the characteristics of the UPS in microtubules in the response of plants to other developmental and environmental cues.

Microtubules provide the trajectories for cellulose synthase complexes in the plasma membrane and guide the deposition direction of cellulose microfibrils to support plant cell growth. This mechanism has been well investigated in both roots and hypocotyls ${ }^{33,34}$. Cellulose also undergoes significant reorganization during stomatal movement from a relatively uniform distribution in the open state to bundles in the closed state ${ }^{35}$. However, the relationship between cellulose reorganization and cortical microtubules during stomatal movement is still unclear. We found that the average angles of the microtubules, which are relative to the stomata inner surface, were reduced in WT but not in rpn1a-4 mutant, WDL7-overexpressing, or mrel57-1 mutant guard cells after ABA treatment (Supplementary Fig. 15). Whether microtubule reorganization affects the arrangement of cellulose, which facilitates repetitive cell wall expansion and contraction during stomatal movement, will be an interesting question to be answered in future work.

Our study indicated that WDL7 has a negative role in UPSmediated ABA-regulated stomatal closure. We did not observe an obvious phenotype for ABA-induced stomatal closure in wdl7 mutants. Although WDL3, a close homolog of WDL7 in WVD2/ WDL family, is involved in ABA-induced stomatal closure, their regulatory mechanisms are quite different, suggesting that the lack of a stomatal closure phenotype in the $w d l 7$ mutants maybe not due to redundancy between WDL7 and other WVD2/WDL family members but due to the degradation of WDL7 protein in response to $\mathrm{ABA}$. This phenomenon has been well reported in some physiological processes involving E3 ligases. For example, 


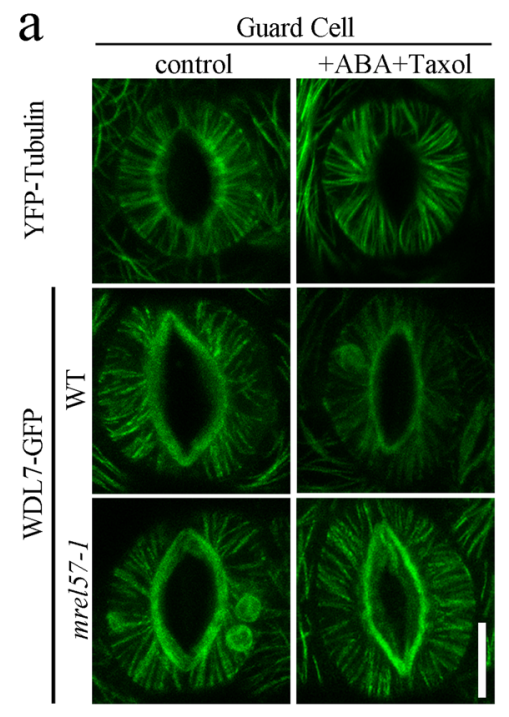

C
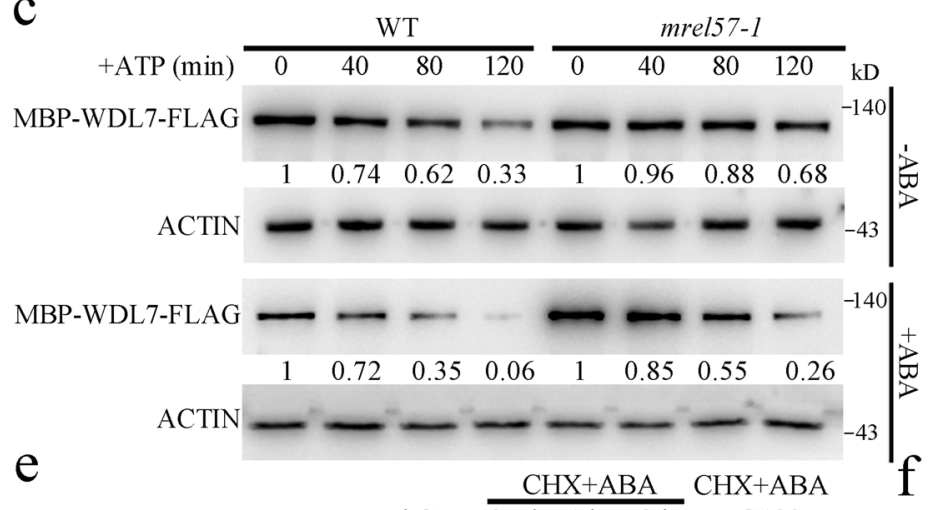

e WT control CHX $\frac{\mathrm{CHX}+\mathrm{ABA}}{0.5 \mathrm{~h} \quad 1 \mathrm{~h} \quad 2 \mathrm{~h}}+\mathrm{CHX}+\mathrm{ABA}$
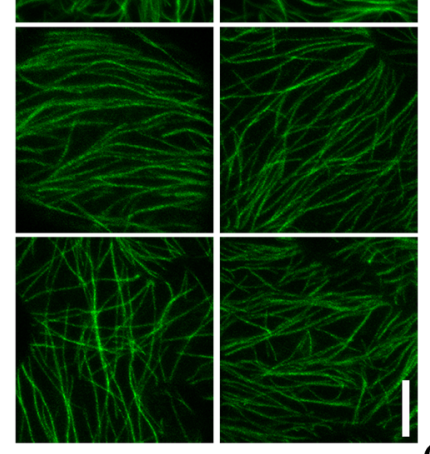

$\mathrm{b}$

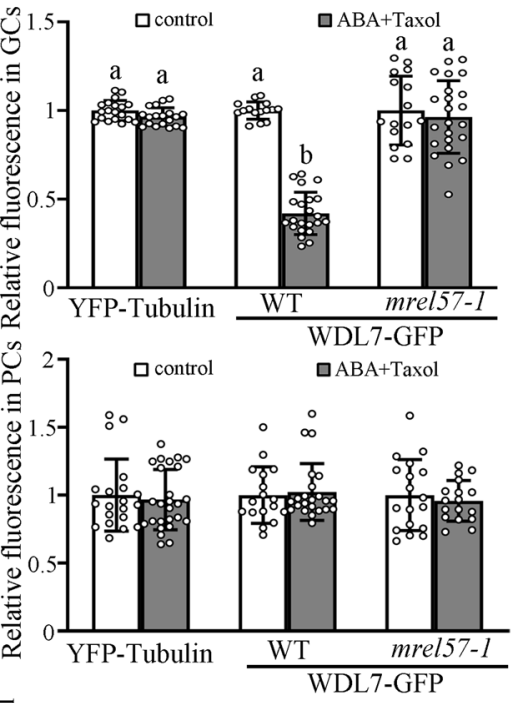

$\mathrm{d} \overline{\mathrm{s}}$
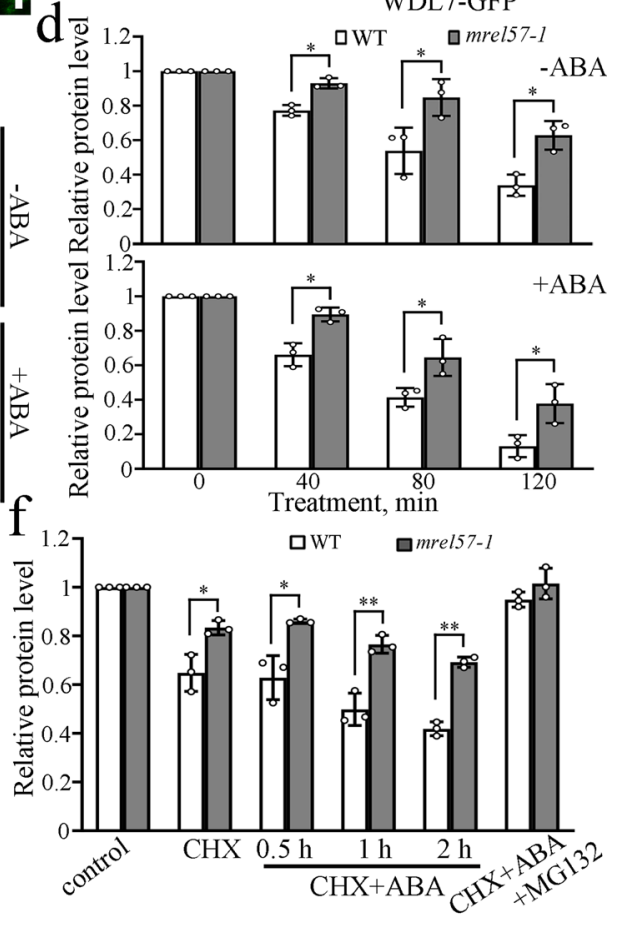

ACTIN
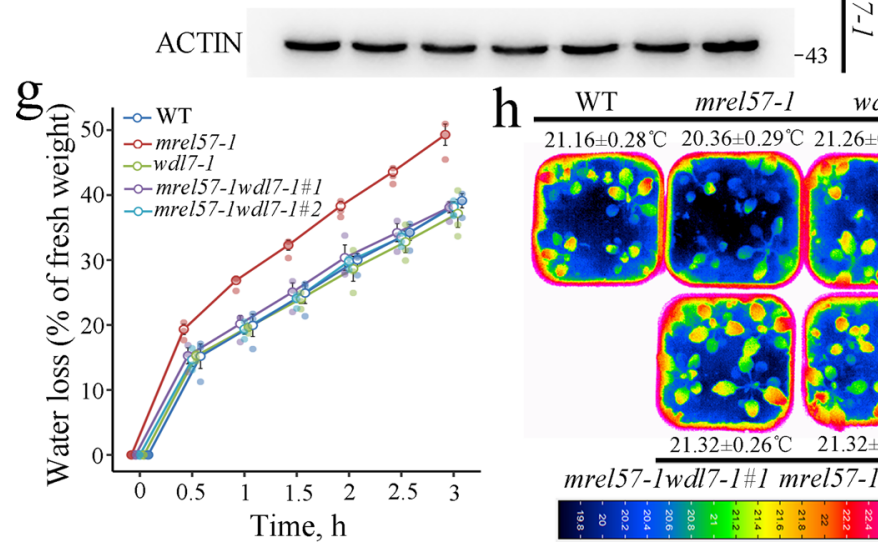

$\mathrm{h}$

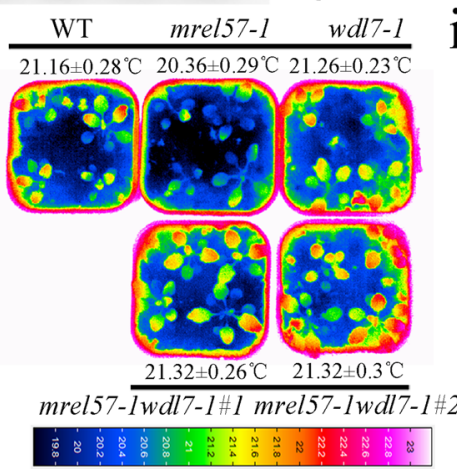

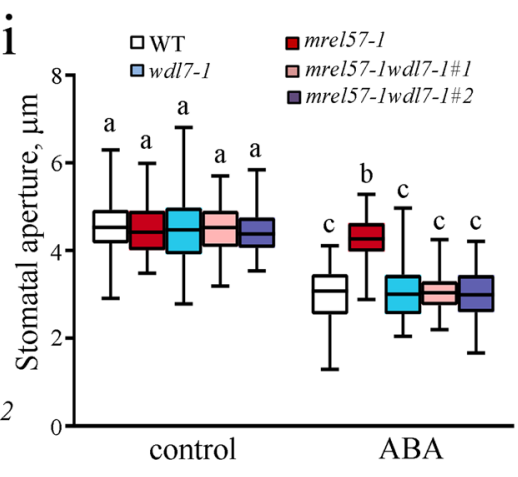

COLD-REGULATED GENE 27 (COR27) and COR28 are negative regulators of photomorphogenesis, and they undergo COP1mediated degradation to avoid too long hypocotyls in darkness. Thus, dark-grown cor 27 cor 28 double mutant seedlings exhibited no hypocotyl-elongated phenotype owing to the degradation of COR27 and COR28 ${ }^{36}$. Our study showed that WDL7 degradation facilitated microtubule disassembly and ABA-induced stomatal closure. Further work is necessary to determine if WDL7 is required in general for stomatal movement, including in response to other environmental cues, such as changes in atmospheric $\mathrm{CO}_{2}$, and temperature.

In this study, we showed that MREL57 and WDL7 transcripts are not regulated by $\mathrm{ABA}$ but that the interaction and ubiquitination of MREL57 with WDL7 were clearly enhanced by ABA, 
Fig. 7 Degradation of WDL7 is required for MREL57-mediated stomatal closure in response to ABA. a Detached rosette leaves of seedlings from YFPtubulin, WDL7-GFP, and WDL7-GFP/mrel57-1 were incubated in opening buffer for $2 \mathrm{~h}$ and then treated with $10 \mu \mathrm{M}$ ABA plus $20 \mu \mathrm{M}$ Taxol for 40 min. Confocal images of the guard cells and pavement cells were taken. Scale bar $=10 \mu \mathrm{m}$. $\mathbf{b}$ The graphs show the relative fluorescence in $\mathbf{a}$. Data represent mean \pm standard deviation (SD) values from three independent experiments with a minimum of 15 cells each. Different letters represent significant differences at $p<0.01$ (one-way ANOVA). c Degradation of WDL7 was clearly decreased in mrel57-1 mutant in a cell-free degradation assay. Purified MBPWDL7-FLAG was incubated with equal amounts of total proteins from 10-day-old WT and mrel57-1 seedlings. MBP-WDL7-FLAG was detected with antiFLAG antibody. Actin was used as a control. d Quantitative analysis of protein levels in $\mathbf{c}$. The WDL7 protein level at $0 \mathrm{~h}$ was set to 1 as a reference for calculating the relative protein levels at the various time points. Data represent the mean \pm SD for three independent experiments. Two-tailed Student's $t$

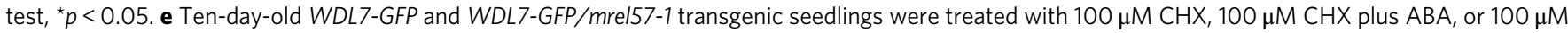
$\mathrm{CHX}$ plus $\mathrm{ABA}$ and MG132 for the indicated times and then total protein was extracted from the leaves. WDL7-GFP was detected with an anti-GFP antibody. Actin was used as a control. $\mathbf{f}$ Quantitative analysis of the protein levels in $\mathbf{e}$. The WDL7 protein level in the leaves from mock buffer-treated seedlings was set to 1 as a reference for calculating the relative protein levels at the various time points. Data represent the mean \pm SD for three independent experiments. Two-tailed Student's $t$ test, ${ }^{\star} p<0.05$, ${ }^{\star \star} p<0.01$. g, Fresh weights of detached leaves of seedlings from WT, mrel57-1, wdl7-1, mrel57-1wdl7-1\#1, and mrel57-1wdl7-1\#2 were measured every $30 \mathrm{~min}$ over a total of $3 \mathrm{~h}$. The experiment was repeated three times with independent treatments. Error bars indicate standard deviation. $\mathbf{h}$ Infrared thermography of seedlings from WT, mrel57-1, wdl7-1, mrel57-1wd/7-1\#1, and mrel57-1wd17-1\#2. Leaf temperature was measured using infrared camera software. Data represent mean \pm standard deviation (SD) values. $\mathbf{i}$ Detached rosette leaves of seedlings from WT, mrel57-1, wdl7-1, mrel57-1wdl7-1\#1, and mrel57-1wdl7-1\#2 were incubated in opening buffer for $2 \mathrm{~h}$ and then treated with $10 \mu \mathrm{M}$ ABA for $2 \mathrm{~h}$. The box and whiskers plots represent minimum and maximum values. The line in the box indicates the median value and the boundaries demonstrate the 25th percentile (upper) and the 75th percentile (lower). Different letters represent significant differences at $p<0.01$ (one-way ANOVA). The experiment was repeated three times as different biological replicates with a minimum of 100 stomatal pores.

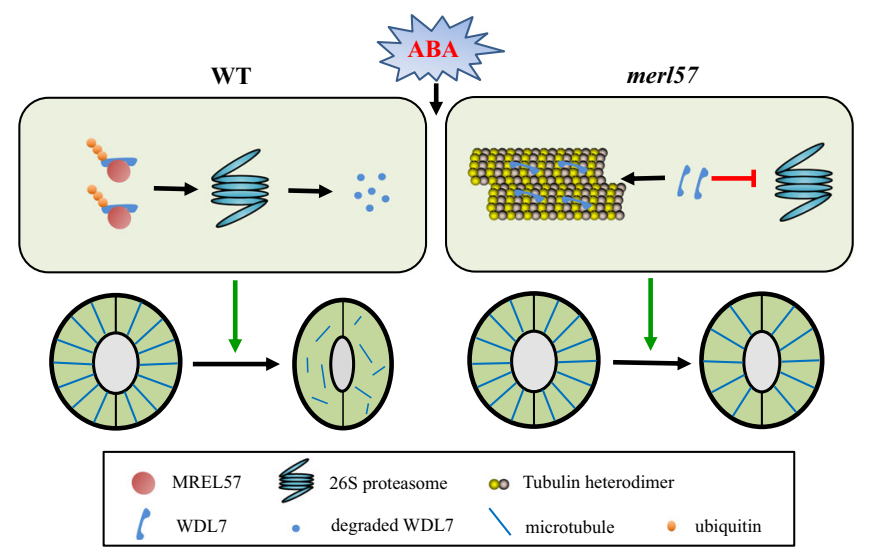

Fig. 8 A hypothetical model for MREL57-WDL7 module function in stomatal closure in response to ABA. Working model for the role of WDL7 proteolysis in ABA-induced stomatal closure and microtubule disassembly in WT and mrel57 mutant guard cells. WDL7 is a microtubule-stabilizing protein. In WT, the E3 ligase MREL57 ubiquitinates and degrades WDL7 in response to $A B A$, which facilitates microtubule disassembly and stomatal closure. In mrel57 mutant, the degradation of WDL7 is blocked. WDL7 binds to and stabilizes microtubules, resulting in stomatal closure insensitivity to $A B A$ in mrel57 mutant.

suggesting that ABA is critical for regulating the activities of MREL57. This phenomenon has been broadly studied in multiple physiological processes involving E3 ligases. For example, SnRK2.6/OST1 kinase phosphorylates and enhances E3 ligase CHYR1 activity during ABA-induced stomatal closure ${ }^{5}$. We found that WDL7 degradation was significantly suppressed in abscisic acid insensitive 1-1 (abil-1) mutants (Supplementary Fig. 16a). Bioinformatics analysis further showed the presence of some potential OST1 phosphorylation motifs in the aminoacid sequence of MREL57 (http://phosphat.uni-hohenheim.de/ phosphat.html? code=AT5G24870.1). Moreover, we found that OST1 interacts with MREL57, and WDL7 degradation was much slower in the ost1-3 mutants (Supplementary Fig. 16b, c). These findings suggest that ABA might regulate the function of the MREL57-WDL7 module through its signaling pathway. Our data indicated that the MREL57-WDL7 module mainly functions in the leaves in response to ABA treatment. Previous studies showed that mesophyll ABA restrains early growth and flowing under non-stress conditions, allowing plants to accumulate biomass and maximize yield ${ }^{37}$. Moreover, mesophyll cells produce significant amounts of ABA under water deficit ${ }^{38}$. Our western blotting results suggested that MREL57 might also interact with and degrade WDL7 in mesophyll cells; however, the role and detailed mechanisms of the MREL57-WDL7 module with ABA treatment in this cell type requires further investigation. In this study, we obtained strong evidence that the MREL57-WDL7 module functions in ABA-induced stomatal closure. These findings provide a new perspective on the cellular control of stomatal movement and further our understanding of the molecular basis for the effects of ABA on the responses of plants to environmental and stressful conditions.

\section{Methods}

Plant materials and growth conditions. The WT ecotype used in this study was A. thaliana Columbia ( $\mathrm{Col})$ and all plant materials were from the Col background. Seeds were sown on $1 / 2$ MS medium (Sigma-Aldrich) with $0.8 \%$ agar and $1 \%$ sucrose $(\mathrm{w} / \mathrm{v})$. The 10-day-old seedlings were transferred into soil and were grown under a 16-h/8-h light/dark photoperiod in a growth room at $22^{\circ} \mathrm{C}$. $35 S$ : YFPtubulin5A transgenic plants, 35S: mCherry-tubulin5A transgenic plants, $35 \mathrm{~S}$ : WDL3-GFP transgenic plants, Pro $_{W D L 3}$ : GUS transgenic plants, rpn1a-4, rpn10-1, abi1-1, ost 1-3, wdl3-1, and wdl5-1 were reported previously $7,21,26,27,39-42$. T-DNA insertion mutants, $w d l 7-1$ (SALK_081293), mrel57-1 (SALK_020143), and mrel572 (SALK_015310), were obtained from the Arabidopsis Biological Resource Center. For overexpressing transgenic plants, the coding sequences of WDL7 and MREL57 were inserted into $P C A M B I A 1390$ and $p B I 121$ vectors under the UBQ10 promoter. To generate Pro $_{W D L 4}:$ GUS, Pro $_{W D L}:$ GUS, and Pro $_{M R E L 57}:$ GUS transgenic plants, the promoters of WDL4, WDL7 and MREL57 were inserted into pCAMBIA1391 vector. To complement the phenotype of the mrel57 mutant, the coding sequence and the promoter of MREL57 were amplified and reconstructed into a $P C A M$ $B I A 1300$ vector. Mutant $w d l 7-2$ was generated by an egg cell-specific promotercontrolled CRISPR/Cas9 system ${ }^{28}$. Constructs were transformed into Arabidopsis plants by Agrobacterium (strain GV3101). Homozygous lines were used for subsequent analyses. The 35S: YFP-tubulin5A/rpn1a-4, 35S:YFP-tubulin5A/mrel57-1, 35S:YFP-tubulin5A/wdl7-1, 35S:YFP-tubulin5A/mrel57-1wdl7-1, UBQ:WDL7-GFP/ mrel57-1, UBQ:WDL7-GFP/ost1-3, mrel57-1wdl7-1, UBQ: WDL7-FLAG MREL57GFP, UBQ:WDL7-GFP mCherry-tubulin, UBQ:WDL7-GFP mCherry-

tubulin/mrel57-1 plants were generated by crossing. Primers are listed in Supplementary Table 1 .

Cell-free protein degradation assay. A cell-free protein degradation assay was performed as described previously ${ }^{43}$. Total WT, mrel57 or abil-1 mutant proteins were extracted with degradation buffer $(25 \mathrm{mM}$ Tris- $\mathrm{HCl}, \mathrm{pH} 7.5 ; 10 \mathrm{mM} \mathrm{NaCl}$; $10 \mathrm{mM} \mathrm{MgCl} ; 4 \mathrm{mM}$ phenylmethylsulfonyl fluoride; $5 \mathrm{mM}$ DTT; $10 \mathrm{mM} \mathrm{ATP}$ ). Purified MBP-WDL7-FLAG protein $(100 \mathrm{ng})$ was incubated with $500 \mu \mathrm{g}$ total 
proteins at $23^{\circ} \mathrm{C}$ for the indicated time. The products were subjected to western blotting with anti-FLAG antibody (ABclonal AE005, 1/5000) and anti-actin antibody (ABclonal AC009, 1/5000).

Water loss assay and drought treatment. For a water loss assay with detached leaves, rosette leaves were cut from 4 -week-old plants in soil. The weight of the detached leaves was measured every $0.5 \mathrm{~h}$ over $\sim 3 \mathrm{~h}$. For the drought treatment, 7 -d-old seedlings were transferred to pots containing the same amount of soil for 2 weeks under normal short-day conditions. After each pot absorbed water fully, the plants were subjected to drought conditions by withholding water for 2 weeks ${ }^{44,45}$. All of the pots for each genotype were distributed in the flat randomly and were rotated frequently to minimize the influence from the environment ${ }^{46}$. Plants that maintained well-watered conditions were used as a control.

Stomatal aperture assay. Stomatal aperture assays were performed as previously described $^{45,47}$. To examine ABA-induced stomatal closure, the rosette leaves of 4 -week-old plants in soil were incubated in opening solution (2-(N-morpholino) ethanesulfonic acid; MES buffer: $50 \mathrm{mM} \mathrm{KCl}, 10 \mathrm{mM} \mathrm{CaCl}_{2}$, and $10 \mathrm{mM} \mathrm{MES}$, $\mathrm{pH}$ 6.15) in a growth chamber for $2 \mathrm{~h}$ to completely open the stomata. Then, the rosette leaves were transferred to opening buffer containing 0 and $10 \mu \mathrm{M} \mathrm{ABA}$ for $2 \mathrm{~h}$. To examine light-induced stomatal opening, the rosette leaves of 4-week-old plants in soil were incubated in MES buffer in a dark growth chamber for $4 \mathrm{~h}$ to close the stomata. Then, the rosette leaves were transferred to the light condition for $1 \mathrm{~h}$. The epidermal strips were peeled from rosette leaves and photographed with an OLYMPUS BX51 microscope. Stomatal apertures were measured with ImageJ software.

Infrared thermograph imaging. Thermal imaging was used to monitor leaf temperature as described previously ${ }^{48}$. Four-week-old plants in soil were transferred from high humidity ( $\mathrm{RH} 70 \%$ ) to low humidity ( $\mathrm{RH} 40 \%$ ) growth conditions. The plants were photographed with a thermal imaging camera (VarioCAM ${ }^{\oplus}$ $\mathrm{HD}$ ), and the leaf temperatures measured using InfraTec IRBIS 3 software (http:// www.InfraTec.net/). Plants in three different pots were analyzed for each genotype, with similar results.

Histochemical GUS activity analysis. For expression patterns of WDL7 or MREL57, seedlings grown in $1 / 2$ MS or soil were subjected to GUS staining. To test the histochemical localization of GUS activity in guard cells, detached rosette leaves from 4-week-old plants in soil were used. The epidermal strips were peeled from the rosette leaves and GUS staining was performed. To examine the histochemical localization of GUS activity in hypocotyls, 5-day-old seedlings grown in the dark and 7-day-old seedlings grown in the light were subjected to GUS staining. GUS assays were performed as described by Jefferson et al. ${ }^{49}$. The transgenic plants were incubated in GUS staining buffer $(0.1 \mathrm{M}$ phosphate-buffered saline (PBS), $0.5 \mathrm{mM}$ potassium ferricyanide, $0.5 \mathrm{mM}$ potassium ferrocyanide, $0.1 \%[\mathrm{v} / \mathrm{v}]$ Triton $\mathrm{X}-100$, $2 \mathrm{mM}$ X-Gluc) for $5-8 \mathrm{~h}$ at $37^{\circ} \mathrm{C}$.

RNA extraction and quantitative real-time PCR analysis. Total RNA was extracted from Arabidopsis seedlings with an RNA purification kit (Bio Teke). RNase-free DNase I (TaKaRa) was used to remove genomic DNA and reverse transcription was performed with M-MLV reverse transcriptase (TaKaRa). Quantitative real-time PCR was carried out with an ABI 7500 PCR system (Applied Biosystems) using SYBR Premix Ex Taq (TaKaRa).

Split-luciferase complementation assay. The coding sequences of MREL57 and $M R E L 57 C 486 A$ were cloned into $p C A M B I A-n L u c$ vectors, and the coding sequences of $W D L 7$ and WDL5 were cloned into $p C A M B I A-c L u c$ vectors. The constructed plasmid vectors were transformed into Agrobacterium (strain GV3101) and then infiltrated into Nicotiana benthamiana leaves. LUC signal was detected by a cold charged-coupled camera (Nikon-L936; Andor Tech) after a 2-day cultivation in a growth room.

Yeast two-hybrid assay. For yeast two-hybrid screening, a normalized universal Arabidopsis cDNA library (Cat. no. 630487; Clontech) was used. The yeast twohybrid assay was performed according to the Yeast Protocols Handbook (Clontech). The coding sequences of MREL57, MREL57N, MREL57C, JUL1, MBR2, $T E A R 1$, and TEAR2 were cloned into $P G A D T 7$ vector, and the coding sequences of $W D L 7$ and $W D L 4$ were cloned into $p G B K T 7$ vector. $p G B K-W D L 3$ was described previously ${ }^{7}$. The resulting vectors were co-transformed into yeast strain AH109. Transformed yeast cells were separately sprayed onto 2D synthetic dropout medium (-Trp/-Leu) and 4D selective medium (-Trp/-Leu/-His/-Ade) and incubated at $28^{\circ} \mathrm{C}$ for $2-3$ days.

Semi-in vivo pull-down assay. For a semi-in vivo pull-down assay, MBP or MBPWDL7-FLAG was used to pull-down MREL57-GFP from seedlings. Total proteins were extracted from 10-day-old MREL57-GFP transgenic seedlings with extraction buffer (50 mM Tris-HCl, pH 7.5; $150 \mathrm{mM} \mathrm{NaCl} ; 10 \mathrm{mM} \mathrm{MgCl}_{2} ; 0.1 \%$ NP-40). MBP or MBP-WDL7-FLAG protein retained on the beads was incubated with the extracted proteins for $2 \mathrm{~h}$ at $4{ }^{\circ} \mathrm{C}$ and the beads were then washed three times with PBS buffer. The pulled down proteins were detected by immunoblot analysis using anti-GFP antibody (Roche 11814460001, 1/5000).

In vitro ubiquitination assay. In vitro ubiquitination assays were performed according to the previous description ${ }^{7,50}$. Approximately $1 \mu \mathrm{g}$ of purified MBPMREL57-MYC or MBP was mixed with $100 \mathrm{ng}$ wheat (Triticum aestivum) E1, $250 \mathrm{ng}$ purified Arabidopsis E2 (AtUBC10), $5 \mu \mathrm{g}$ ubiquitin (Boston Biochem, Cat. No. U-100AT), and $500 \mathrm{ng}$ purified MBP-WDL7-FLAG in buffer containing $50 \mathrm{mM}$ Tris- $\mathrm{HCl}$ (pH 7.4), $2 \mathrm{mM}$ ATP, $5 \mathrm{mM} \mathrm{MgCl}_{2}, 2 \mathrm{mM} \mathrm{DTT}$, and $0.05 \mathrm{mM}$ $\mathrm{ZnCl}_{2}$. After incubation at $30^{\circ} \mathrm{C}$ for $3 \mathrm{~h}$, the reactions were stopped with $5 \times$ sodium dodecyl sulfate sample buffer. The reaction products were analyzed by $6 \%$ sodium dodecyl sulphate polyacrylamide gel electrophoresis (SDS-PAGE) and immunoblotted using the appropriate antibodies. Self-ubiquitination of MBP-MREL57MYC was detected with anti-MYC antibody (ABclonal AE010, 1/5000). Ubiquitinated MBP-WDL7-FLAG was detected with anti-FLAG antibody (ABclonal AE005, 1/5000).

In vivo ubiquitination assay. An in vivo ubiquitination assay was performed as described previously ${ }^{51}$. Ten-day-old WDL7-GFP and WDL7-GFP/mrel57-1 transgenic seedlings were treated with $1 / 2$ MS liquid medium supplemented with 0 or $10 \mu \mathrm{M}$ of ABA (Sigma) for $1 \mathrm{~h}$ in the presence of $50 \mu \mathrm{M}$ MG132 (Sigma). Total proteins were extracted with extraction buffer and incubated for $2 \mathrm{~h}$ with anti-GFP mAb-magnetic agarose (MBL, Cat. No. D153-10). The beads were then washed three times with PBS buffer. The proteins were separated on 6\% SDS-PAGE and WDL7-GFP ubiquitination was detected with anti-Ub antibody (Santa Cruz Biotechnology SC8017, 1/2000).

Low-temperature and dilution assays. Low-temperature and dilution assays were performed as described previously 26,27 . His-WDL7-GFP protein $(0$ or $3 \mu \mathrm{M})$ was added to $20 \mu \mathrm{M}$ rhodamine-labeled tubulin in PEM buffer $(1 \mathrm{mM} \mathrm{MgCl}, 1 \mathrm{mM}$ EGTA, and $100 \mathrm{mM}$ PIPES-KOH, pH 6.9) containing $1 \mathrm{mM} \mathrm{GTP}$ and the samples were then incubated at $35^{\circ} \mathrm{C}$ for $40 \mathrm{~min}$ to allow tubulin assembly. For the lowtemperature assay, the temperature was immediately decreased to $10^{\circ} \mathrm{C}$ and maintained for $30 \mathrm{~min}$. For the dilution assay, the assembled tubulin samples described above were diluted with $50 \times$ prewarmed PEM buffer containing HisWDL7-GFP protein $(0$ or $3 \mu \mathrm{M})$ and incubated at $35^{\circ} \mathrm{C}$ for $60 \mathrm{~min}$ prior to fixation. The samples were fixed with $1 \%(\mathrm{v} / \mathrm{v})$ glutaraldehyde for observation under confocal microscopy.

Observation and quantification of cortical microtubules in cells. The rosette leaves of plants were incubated in opening solution (MES buffer) in a growth chamber for $2 \mathrm{~h}$ to open stomata completely. The leaves were then transferred to MES buffer containing different drugs for the indicated time periods. Cortical microtubules in pavement cells and guard cells were observed using a confocal laser scanning microscope (LSM 880, Carl Zeiss). YFP was excited at $488 \mathrm{~nm}$ and mCherry was excited at $561 \mathrm{~nm}$. To preprocess the quantitative evaluations of microtubule density, maximum-intensity projections were constructed from serial optical sections. The images were then binarized by thresholding and skeletonized using the ImageJ menu "Process-Binary-Skeletonize". The density of microtubules was defined as the occupancy of the GFP signal in guard cells ${ }^{23}$. To quantify the extent of microtubule bundling in guard cells, skewness of the intensity distribution of the microtubule pixels was measured as previously described ${ }^{23,52}$. Micrographs were analyzed with ImageJ using Higaki's macro ${ }^{23}$.To evaluate microtubule orientation, the angle of each microtubule to the inner surface of the stomata was measured using ImageJ software ${ }^{23}$. All the analyses were carried out using the 8 bit raw scanning images.

Reporting summary. Further information on research design is available in the Nature Research Reporting Summary linked to this article.

\section{Data availability}

Sequence data of the genes described in this article can be found in the Arabidopsis Genome Initiative under the following accession numbers: At5g24870, MREL57; At1g70950, WDL7; At3g23090, WDL3; At2g35880, WDL4; At4g32330, WDL5; At5g52310, RD29A; At5g52300, RD29B; At2g20580, RPN1A; At4g38630, RPN10; At4g33950, OST1; At4g26080, ABI1; At5g10650, JUL1; At4g34040, MBR2; Atlg53190, TEAR1; At3g15070, TEAR2. The source data underlying Figs. 1b, d, e, 2a-g, 3b-g, 4b, f, g, $5 \mathrm{c}, \mathrm{f}-\mathrm{j}, 6 \mathrm{~b}-\mathrm{h}, 7 \mathrm{~b}-\mathrm{I}$, and Supplementary Figs. 2, 4b-e, 5a-e, 8b, c, 10b, c, 11b, 12b, d, $14 a-c, 15,16 a, c$ are provided in the Source Data file. All data supporting findings of this manuscript are available from the article and Supplementary Information files, or from the corresponding author upon request. Source data are provided with this paper.

Received: 19 March 2020; Accepted: 12 March 2021; Published online: 12 April 2021 


\section{References}

1. Hetherington, A. M. \& Woodward, F. I. The role of stomata in sensing and driving environmental change. Nature 424, 901-908 (2003).

2. Kim, T. H., Böhmer, M., Hu, H., Nishimura, N. \& Schroeder, J. I. Guard cell signal transduction network: advances in understanding abscisic acid, $\mathrm{CO}_{2}$, and $\mathrm{Ca}^{2+}$ signaling. Annu. Rev. Plant Biol. 61, 561-591 (2010).

3. Kollist, H., Nuhkat, M. \& Roelfsema, M. R. Closing gaps: linking elements that control stomatal movement. N. Phytol. 203, 44-62 (2014).

4. Agurla, S. \& Raghavendra, A. S. Convergence and divergence of signaling events in guard cells during stomatal closure by plant hormones or microbial elicitors. Front. Plant Sci. 7, 1332 (2016).

5. Ding, S., Zhang, B. \& Qin, F. Arabidopsis RZFP34/CHYR1, a ubiquitin E3 ligase, regulates stomatal movement and drought tolerance via SnRK2.6mediated phosphorylation. Plant Cell 27, 3228-3244 (2015).

6. Wang, X. L. et al. Arabidopsis MICROTUBULE DESTABILIZING PROTEIN40 is involved in brassinosteroid regulation of hypocotyl elongation. Plant Cell 24, 4012-4025 (2012).

7. Lian, N. et al. COP1 mediates dark-specific degradation of microtubuleassociated protein WDL3 in regulating Arabidopsis hypocotyl elongation. Proc. Natl Acad. Sci. USA 46, 12321-12326 (2017).

8. Dou, L. R., He, K. K., Higaki, T., Wang, X. F. \& Mao, T. L. Ethylene signaling modulates cortical microtubule reassembly in response to salt stress. Plant Physiol. 176, 2071-2081 (2018).

9. Wang, X. F. \& Mao, T. L. Understanding the functions and mechanisms of plant cytoskeleton in response to environmental signals. Curr. Opin. Plant Biol. 52, 86-96 (2019).

10. Nick, P. Microtubules, signalling and abiotic stress. Plant J. 75, 309-323 (2013).

11. Lindeboom, J. J. et al. A mechanism for reorientation of cortical microtubule arrays driven by microtubule severing. Science 342, 1245533 (2013).

12. Kesten, C. et al. The companion of cellulose synthase 1 confers salt tolerance through a Tau-like mechanism in plants. Nat. Commun. 10, 857 (2019).

13. Assmann, S. M. \& Baskin, T. I. The function of guard cells does not require an intact array of cortical microtubules. J. Exp. Bot. 49, 163-170 (1998).

14. Marcus, A. I., Moore, R. C. \& Cyr, R. J. The role of microtubules in guard cell function. Plant Physiol. 125, 387-395 (2001).

15. Eisinger, W., Ehrhardt, D. \& Briggs, W. Microtubules are essential for guardcell function in Vicia and Arabidopsis. Mol. Plant 5, 601-610 (2012).

16. Lahav, M., Abu-Abied, M., Belausov, E., Schwartz, A. \& Sadot, E. Microtubules of guard cells are light sensitive. Plant Cell Physiol. 45, 573-582 (2004).

17. Jiang, Y. et al. Phosphatidic acid integrates calcium signaling and microtubule dynamics into regulating ABA-induced stomatal closure in Arabidopsis. Planta 239, 565-575 (2014).

18. Hershko, A. \& Ciechanover, A. The ubiquitin system. Annu. Rev. Biochem. 67, 425-479 (1998).

19. Vierstra, R. D. The ubiquitin-26s proteasome system at the nexus of plant biology. Nat. Rev. Mol. Cell Biol. 10, 385-397 (2009).

20. Sadanandom, A., Bailey, M., Ewan, R., Lee, J. \& Nelis, S. The ubiquitinproteasome system: central modifier of plant signalling. N. Phytol. 196, 13-28 (2012).

21. Wang, S., Kurepa, J., Hashimoto, T. \& Smalle, J. A. Salt stress-induced disassembly of Arabidopsis cortical microtubule arrays involves 26S proteasome-dependent degradation of SPIRAL1. Plant Cell 23, 3412-3427 (2011).

22. Khanna, R. et al. COP1 jointly modulates cytoskeletal processes and electrophysiological responses required for stomatal closure. Mol. Plant 7, 1441-1454 (2014).

23. Higaki, T., Kutsuna, N., Sano, T., Kondo, N. \& Hasezawa, S. Quantification and cluster analysis of actin cytoskeletal structures in plant cells: role of actin bundling in stomatal movement during diurnal cycles in Arabidopsis guard cells. Plant J. 61, 156-165 (2010)

24. Wang, C., Li, J. \& Yuan, M. Salt tolerance requires cortical microtubule reorganization in Arabidopsis. Plant Cell Physiol. 48, 1534-1547 (2007).

25. Kuki, H. et al. Quantitative confocal imaging method for analyzing cellulose dynamics during cell wall regeneration in Arabidopsis mesophyll protoplasts. Plant Direct 1, e00021 (2017).

26. Liu, X. M. et al. Light-regulated hypocotyl elongation involves proteasomedependent degradation of the microtubule regulatory protein WDL3 in Arabidopsis. Plant Cell 25, 1740-1755 (2013).

27. Sun, J. B., Ma, Q. Q. \& Mao, T. L. Ethylene regulates the Arabidopsis microtubule-associated protein WAVE-DAMPENED2-LIKE 5 in etiolated hypocotyl elongation. Plant Physiol. 169, 325-337 (2015)

28. Wang, Z. P. et al. Egg cell-specific promoter-controlled CRISPR/Cas9 efficiently generates homozygous mutants for multiple target genes in Arabidopsis in a single generation. Genome Biol. 16, 144 (2015).
29. Perrin, R. M., Wang, Y., Yuen, C. Y., Will, J. \& Masson, P. H. WVD2 is a nove microtubule-associated protein in Arabidopsis thaliana. Plant J. 49, 961-971 (2007).

30. Mao, T. L., Jin, L. F., Li, H., Liu, B. \& Yuan, M. Two microtubule-associated proteins of the Arabidopsis MAP65 family function differently on microtubules. Plant Physiol. 138, 654-662 (2005).

31. Kosarev, P., Mayer, K. F. \& Hardtke, C. S. Evaluation and classification of RING-finger domains encoded by the Arabidopsis genome. Genome Biol. 3, 1-12 (2002)

32. Chen, H. et al. Firefly luciferase complementation imaging assay for protein protein interactions in plants. Plant Physiol. 146, 368-376 (2008).

33. Gutierrez, R., Lindeboom, J. J., Paredez, A. R., Emons, A. M. \& Ehrhardt, D W. Arabidopsis cortical microtubules position cellulose synthase delivery to the plasma membrane and interact with cellulose synthase trafficking compartments. Nat. Cell Biol. 11, 797-806 (2009).

34. Bashline, L., Li, S. \& Gu, Y. The trafficking of the cellulose synthase complex in higher plants. Ann. Bot. 114, 1059-1067 (2014)

35. Rui, Y. \& Anderson, C. T. Functional analysis of cellulose and xyloglucan in the walls of stomatal guard Cells of Arabidopsis. Plant Physiol. 170, 1398-1419 (2016)

36. $\mathrm{Li}, \mathrm{X}$. et al. COR27 and COR28 are novel regulators of the COP1-HY5 regulatory hub and photomorphogenesis in Arabidopsis. Plant Cell 32, 3139-3154 (2020).

37. Negin, B., Yaaran, A., Kelly, G., Zait, Y. \& Moshelion, M. Mesophyll abscisic acid restrains early growth and flowering but does not directly suppress photosynthesis. Plant Physiol. 180, 910-925 (2019).

38. McAdam, S. A. M. \& Brodribb, T. J. Mesophyll cells are the main site of abscisic acid biosynthesis in water-stressed leaves. Plant Physiol. 177, 911-917 (2018).

39. Kirik, A., Ehrhardt, D. W. \& Kirik, V. TONNEAU2/FASS regulates the geometry of microtubule nucleation and cortical array organization in interphase Arabidopsis cells. Plant Cell 24, 1158-1170 (2012).

40. Kong, L. Y. et al. Degradation of the ABA co-receptor ABI1 by PUB12/13 U box E3 ligases. Nat. Commun. 6, 8630 (2015)

41. Yang, Y. Q. et al. Arabidopsis IPGA1 is a microtubule-associated protein essential for cell expansion during petal morphogenesis. J. Exp. Bot. 70, 5231-5243 (2019).

42. Ding, Y. L. et al. OST1 kinase modulates freezing tolerance by enhancing ICE1 stability in Arabidopsis. Dev. Cell 32, 278-289 (2015).

43. Zhang, M. et al. The Arabidopsis U-box E3 ubiquitin ligase PUB30 negatively regulates salt tolerance by facilitating BRI1 kinase inhibitor 1 (BKI1) degradation. Plant Cell Environ. 40, 2831-2843 (2017)

44. Shi, H. T., Ye, T. T., Yang, F. \& Chan, Z. L. Arabidopsis PED2 positively modulates plant drought stress resistance. J. Integr. Plant Biol. 57, 796-806 (2015).

45. Wang, $\mathrm{K}$. et al. EAR1 negatively regulates ABA signaling by enhancing $2 \mathrm{C}$ protein phosphatase activity. Plant Cell 30, 815-834 (2018).

46. Chen, J. N. et al. Arabidopsis WRKY46, WRKY54, and WRKY70 transcription factors are involved in brassinosteroid-regulated plant growth and drought responses. Plant Cell 29, 1425-1439 (2017)

47. Xue, Y., Yang, Y. Q., Yang, Z. J., Wang, X. F. \& Guo, Y. VAMP711 is required for abscisic acid-mediated inhibition of plasma membrane $\mathrm{H}^{+}$-ATPase activity. Plant Physiol. 3, 1332-1343 (2018).

48. Xie, $\mathrm{X}$. et al. The identification of genes involved in the stomatal response to reduced atmospheric relative humidity. Curr. Biol. 16, 882-887 (2006)

49. Jefferson, R. A., Kavanagh, T. A. \& Bevan, M. W. GUS fusions: betaglucuronidase as a sensitive and versatile gene fusion marker in higher plants. EMBO J. 6, 3901-3907 (1987).

50. Xiao, Z. D. et al. SINAT E3 ligases regulate the stability of the ESCRT component FREE1 in response to iron deficiency in plants. J. Integr. Plant Biol. 62, 1399-1417 (2020).

51. Wang, X. et al. PUB25 and PUB26 promote plant freezing tolerance by degrading the cold signaling negative regulator MYB15. Dev. Cell 51, 1-14 (2019).

52. Ma, Q. Q., Sun, J. B. \& Mao, T. L. Microtubule bundling plays a role in ethylene-mediated cortical microtubule reorientation in etiolated Arabidopsis hypocotyls. J. Cell Sci. 129, 2043-2051 (2016).

\section{Acknowledgements}

The authors appreciate the funding support received from the National Science Fund for Distinguished Young Scholars (31625005 to T.M.), the Natural Science Foundation of China (31872821 to T.M. and 31872644 to X.W.), and China Postdoctoral Science Foundation (2019M660868 and 2019TQ0352 to L.D.). The authors thank Zhizhong Gong (China Agricultural University) and Jiejie Li (Beijing Normal University) for comments on the article. 


\section{Author contributions}

L.D. and T.M. designed the experiments; L.D., K.H., and J.P. performed the experiments; L.D., K.H., X.W., and T.M. analyzed the data and wrote the paper. All authors read and approved the paper.

\section{Competing interests}

The authors declare no competing interests.

\section{Additional information}

Supplementary information The online version contains supplementary material available at https://doi.org/10.1038/s41467-021-22455-y.

Correspondence and requests for materials should be addressed to T.M.

Peer review information Nature Communications thanks Takumi Higaki, Vicente Rubio, and the other, anonymous reviewer(s) for their contribution to the peer review of this work.
Reprints and permission information is available at http://www.nature.com/reprints

Publisher's note Springer Nature remains neutral with regard to jurisdictional claims in published maps and institutional affiliations.

(c) Open Access This article is licensed under a Creative Commons Attribution 4.0 International License, which permits use, sharing, adaptation, distribution and reproduction in any medium or format, as long as you give appropriate credit to the original author(s) and the source, provide a link to the Creative Commons license, and indicate if changes were made. The images or other third party material in this article are included in the article's Creative Commons license, unless indicated otherwise in a credit line to the material. If material is not included in the article's Creative Commons license and your intended use is not permitted by statutory regulation or exceeds the permitted use, you will need to obtain permission directly from the copyright holder. To view a copy of this license, visit http://creativecommons.org/licenses/by/4.0/.

(c) The Author(s) 2021 